\title{
Multibody model based estimation of multiple loads and strain field on a vehicle suspension system
}

\author{
Enrico Risaliti $^{\dagger, a, b}$, Tommaso Tamarozzi ${ }^{\mathrm{a}, \mathrm{b}}$, Martijn Vermaut $^{\mathrm{b}, \mathrm{c}}$, Bram Cornelis $^{\mathrm{a}}$, Wim Desmet $^{\mathrm{b}, \mathrm{c}}$ \\ ${ }^{a}$ Siemens PLM Software, Interleuvenlaan 68, 3001 Leuven (Belgium) \\ ${ }^{b}$ Katholieke Universiteit Leuven, Celestijnenlaan 300 B, 3001, Heverlee (Belgium) \\ ${ }^{c}$ DMMS core lab, Flanders Make (Belgium) \\ $\dagger$ Corresponding author, e-mail: enrico.risaliti@siemens.com
}

\begin{abstract}
This work proposes an augmented extended Kalman filter based state-input estimator for mechanical systems defined by implicit equations of motion which is then applied to estimate the six wheel center loads and the strain field on a vehicle suspension test rig.

Implicit equations of motion typically arise in the definition of flexible multibody models and also in their time resolution, because implicit time-discretization schemes are normally employed to obtain a stable solution. The presented methodology can be applied to such case and analytical expressions are derived for the necessary linearizations, providing the means for a computationally efficient estimation procedure.

The six wheel center loads and the strain field on a vehicle suspension system are valuable quantities during the vehicle design phase (e.g. for durability analysis), hence they are often directly measured during elaborate full vehicle testing campaigns. This work demonstrates that a flexible multibody model representation allows to accurately reconstruct the time domain signals of the six loads and of the full strain field, starting from a minimal set of six measured strains, hence providing an appealing alternative to direct measurement methods. The experimental validation on the suspension test rig shows that all estimated quantities can be accurately reconstructed, given that the system simulation model incorporates an adequate level of accuracy.
\end{abstract}

Keywords: Multiple load estimation, State estimation, Multibody dynamics, Virtual sensing, Automotive durability engineering.

\section{Introduction}

When designing a new vehicle, the knowledge of Wheel Center Loads (WCLs) is needed as an input quantity to assess durability performances [1]. In the current practice Original Equipment Manufacturers (OEMs) perform direct measurements of these loads during so-called Road Load Data Acquisition (RLDA) testing campaigns in which a prototype vehicle is heavily instrumented and then driven on proving grounds or public roads. The instrumentation includes so-called Wheel Force Transducers (WFTs) which directly measure WCLs, but which turn out to be expensive and intrusive. Therefore it is appealing to find an alternative way of measuring WCLs which does not make use of such measurement devices.

During RLDA up to hundreds of other sensor channels including strain gauges, accelerometers and displacement sensors are acquired along with WFTs channels. On the other hand, virtual prototypes, i.e. system simulation models, are more and more used to assess the vehicle performances already before the first real prototype is built and tested. Therefore an appealing alternative is to re-use the readily available system simulation models in combination with these other sensors, in order to virtually measure the WCLs following the analysis paradigm outlined in [2]. This would imply a cost reduction and hence a tangible increase of the overall process efficiency.

A state estimation framework is developed in this work to perform the information fusion between the available measurements and a flexible multibody model of the vehicle suspension system. Such type of system simulation model is needed to accurately reconstruct the time domain signal for all six WCLs. In addition, this also allows to virtually measure the full strain field on the suspension flexible components. 


\subsection{Indirectly measuring the inputs of a mechanical system}

The question of indirectly measuring the inputs of a mechanical system is a particular case of inverse problem which has been widely investigated by researchers over the decades. A review of the major developments in inversion of linear systems can be found in [3]. Alternative methods, namely the so-called deconvolution methods, are instead proposed in [4] and [5], while a review of frequency domain methods can be found in [6]. Of the previously mentioned approaches, only some can reconstruct both inputs and states of the system, while the reconstruction of states is also important in this work. Indeed, the reconstruction of system outputs at unmeasured locations, which can be performed starting from the system states, is also envisioned. Kalman Filters (KFs) are the most widespread and successfully used state estimators. Those have been initially developed in [7] to estimate the states of systems of which the inputs are known. However several extensions to estimate unknown inputs along with the system states have been proposed over the years. The simplest approach consists in augmenting the system state vector with the unknown inputs and in introducing a so-called random walk model for the unknown inputs while employing a standard Kalman Filter. This leads to the so-called Augmented Kalman Filter (AKF) which has been recently used in structural dynamics problems in [8], [9] and [10]. A slightly different approach still assumes a random walk model for the unknown inputs, but performs the estimation of the unknown inputs and of the system states in two sequential steps inside a classical Kalman Filter scheme. Such approach, which can be found in [11] and has been first applied to structural dynamics problems in [12], is commonly referred to as the Dual Kalman Filter (DKF). In [13] a joint input-state estimator has been proposed, in which an additional model for the unknown inputs does not need to be introduced. The resulting scheme is similar to the regular Kalman Filter, with the difference that the inputs true values are replaced with their optimal estimates obtained in a minimum-variance sense. Such an approach, to which we refer herewith with Joint Input-State Estimator (JISE), has been also extended to systems with direct feedthrough in [14] and successively applied to structural dynamics problems in [15] and [16].

Although all the methods and applications mentioned above are limited to linear dynamic systems, methods to deal with non-linear problems have been also developed over the years. The well-known Extended Kalman Filter (EKF), which can be found in [17], is one of the most popular ones and derives from the application of the linear KF to a linearization of the non-linear system equations. If the system exhibits strong non-linearities in relation to the sampling rate, the quality of the estimation with the EKF can be deteriorated by the linearization procedure. In such cases the so-called Unscented Kalman Filter (UKF) introduced in [18] can outperform the EKF, as it makes use of the original non-linear transformation on so-called sigma points to estimate the state statistical moments. However as shown in [19], little difference can be observed between the results of the EKF and the UKF in the case of weakly nonlinear systems. Furthermore if the system linearization is analytically available, the EKF becomes computationally more efficient, whereas the computation of sigma points represents a computational burden when dealing with systems of medium to large sizes. For this reason the EKF is preferred in this work, as it is assumed that analytical expressions for the system linearizations are available.

Both an Augmented and a Dual form of the aforementioned non-linear Kalman Filters, i.e. non-linear counterparts of the AKF and of the DKF, can be easily obtained, whereas the extension of the JISE to non-linear estimation problems is not trivial. A step in this sense has been made in [20] in which a generalized minimum variance unbiased joint input-state estimation embedded in an UKF scheme has been developed but only for systems with direct feedthrough. The ease of derivation of the Augmented form makes the Augmented Extended Kalman Filter (AEKF) the method of choice in this work, while the Dual Extended Kalman Filter (DEKF) is left as possible part of future work.

\subsection{Dealing with stiff and implicit mechanical problems}

A number of works related to estimation with multibody systems have also been proposed, for which non-linear estimators are needed. In [21] a continuous-time version of the EKF is combined with the multibody dynamics equations in order to estimate the states of the system, whereas in [22] an UKF has been adopted for the same scope. In [23] an AEKF is proposed to estimate both states and inputs of a reduced order multibody model. A two-stage approach is presented in [24], where a so-called Kinematic Kalman Filter (KKF) based on the kinematic constraint equations estimates the states of the system while a nonlinear Kalman filter based on the multibody dynamic equations and a random walk model is used to estimate unknown inputs. In [25] an AEKF is adopted in a simulated example to estimate the states and a single input in a suspension system modeled as a multibody system. In [26] a so-called Error Extended Kalman Filter (EEKF), in which the state error with respect to the true value is estimated in the place of the 
state itself, is used in combination with multibody models. In the same work a random walk model is introduced in order to estimate the acceleration vector and subsequently the unknown inputs.

In all the aforementioned works related to estimation problems for multibody systems, examples related to simple mechanisms composed of rigid bodies have been given. This work instead deals with flexible multibody systems and hence particular attention is paid to obtaining an estimation procedure which is stable with respect to the numerical stiffness associated with the time-discretization of the system equations in the sense intended in [27]. Such computational aspects have been taken under considerations in [28], [29] and [30] when dealing with a continuous-time version of the EKF. However, when adopting a continuous-time EKF additional precautions have to be adopted in order to guarantee that the employed time-discretization scheme leads to a stable estimation procedure as pointed out in [29] and for this reason a discrete-time version of the EKF is preferred in this work. An implicit time-discretization scheme is then used in order to cope with the numerical stiffness of the problem.

In addition, the EKF has been traditionally developed for systems that can be described by an explicit state equation, which might not be the case for multibody systems if particular choices of the generalized coordinates used to describe the system configuration are made, as in [31] and [32]. In such cases only an implicit state equation is available even before the time-discretization step is performed.

Both the need for an implicit time-discretization scheme or the absence of an explicit description of the state equation on continuous-time level cause having to derive an EKF for implicit discrete-time state equations. A few examples in which EKFs have been applied to implicitly described systems can be found in [33] and [34]. In those examples however some structure of the equations is assumed. In this work instead, a general procedure is presented to derive an EKF and an AEKF for systems described by an implicit state equation of general form.

\subsection{Main contributions and paper outline}

Summarizing, a methodology is proposed to derive an input-state estimation procedure based on an AEKF for stiff mechanical systems described by an implicit state equation. This extends the applicability of current frameworks for input-state estimation to e.g. flexible multibody systems. The methodology is then applied to a real experiment which is representative of an industrial application, i.e. RLDA testing campaigns, for the estimation of multiple loads and of the full strain field of the structure sub-components. In such example the system is described as a flexible multibody system, which represents a particular case of an implicitly defined stiff mechanical system. Additionally, an optimal sensor selection procedure which makes use of the available non-linear system model is employed.

The paper is organized as follows. In section 2 the proposed methodology is presented. In section 3 the system under investigation for the experimental validation, i.e. a quarter car test rig based on a McPherson suspension system, is described. In section 4 the employed sensor sets are described along with the strategy adopted for their definition. Section 5 shows the validation results, while the conclusions of the work are presented in section 6.

\section{Discrete Extended Kalman Filtering of implicit and stiff non-linear mechanical problems}

This paper addresses the problem of estimating the states of a dynamic system described by the following continuoustime state space equation

$$
\mathbf{g}(\dot{\mathbf{x}}, \mathbf{x}, \mathbf{u})=\mathbf{0}
$$

where $\mathbf{g}$ is a real-valued non-linear function, $\mathbf{x} \in \mathbb{R}^{n_{S}}$ is the state vector and $\mathbf{u} \in \mathbb{R}^{n_{I}}$ is the input vector. The governing Eq. 1 is implicit in all its variables, including the time derivative of the state vector $\dot{\mathbf{x}}$. The majority of optimal estimation problems are instead formulated, since the first formulation in [7], for dynamic systems that can be described by the following continuous-time explicit state space equation

$$
\dot{\mathbf{x}}=\mathbf{f}(\mathbf{x}, \mathbf{u})
$$

where $\mathbf{f}$ is also a real-valued non-linear function. For this reason a dedicated procedure for estimating the states of a dynamic system described by Eq. 1 is presented in the following subsections.

The explicit dependency with respect to time of the variables in Eqs. 1 and 2 is omitted as it is omitted from the equations appearing in the rest of the paper. In general all the variables are functions of time, unless they are related to a specific point in time. 


\subsection{The implicit system's Equations of Motion}

The system under investigation is described as a flexible multibody system, which is a set of rigid or flexible interconnected bodies, therefore constraints equations have to be introduced to model the joints between the bodies. This reads

$$
\mathbf{\Phi}(\mathbf{q})=\mathbf{0}
$$

where $\boldsymbol{\Phi}$ is the constraint vectorial function of dimension $n_{c}$ and $\mathbf{q} \in \mathbb{R}^{n_{G C}}$ is the vector of the generalized coordinates. The latter is a set of coordinates which can be used to write the kinetic energy $T(\mathbf{q}, \dot{\mathbf{q}})$ and the potential energy $V(\mathbf{q})$ of the system. The governing Equations of Motion (EOMs) of the system can then be found by means of the Lagrange's equations for constrained mechanical systems [35] as

$$
\frac{d}{d t} \frac{\partial L(\mathbf{q}, \dot{\mathbf{q}})}{\partial \dot{\mathbf{q}}}-\frac{\partial L(\mathbf{q}, \dot{\mathbf{q}})}{\partial \mathbf{q}}+\boldsymbol{\Phi}_{\mathbf{q}}(\mathbf{q})^{T} \lambda=\mathbf{Q}^{e}(\mathbf{q}, \mathbf{u})
$$

where $L$ is the Lagrangian function defined as $L=T-V, \boldsymbol{\Phi}_{\mathbf{q}}$ is the constraints Jacobian matrix defined as $\boldsymbol{\Phi}_{\mathbf{q}}=$ $\partial \mathbf{\Phi} / \partial \mathbf{q}, \lambda \in \mathbb{R}^{n_{C}}$ is the Lagrange multipliers vector and $\mathbf{Q}^{e}$ is the vector of the external actions which depends on the generalized coordinates $\mathbf{q}$ and on the input vector $\mathbf{u}$. $\lambda$ represents an additional set of algebraic variables which can be found along with $\mathbf{q}$ by solving the system of Differential Algebraic Equations (DAEs) represented by Eqs. 4 and 3 . In this work however, a different way is used to incorporate the constraints in the EOMs, that is the term $\boldsymbol{\Phi}_{\mathbf{q}}(\mathbf{q})^{T} \lambda$ of Eq. 4 is replaced with the penalty term $\boldsymbol{\Phi}_{\mathbf{q}}^{T}(\alpha \boldsymbol{\Phi}+\beta \dot{\boldsymbol{\Phi}})$ [35], in which the two penalty factors $\alpha$ and $\beta$ are arbitrary numbers. This leads to

$$
\frac{d}{d t} \frac{\partial L(\mathbf{q}, \dot{\mathbf{q}})}{\partial \dot{\mathbf{q}}}-\frac{\partial L(\mathbf{q}, \dot{\mathbf{q}})}{\partial \mathbf{q}}+\boldsymbol{\Phi}_{\mathbf{q}}^{T}(\alpha \boldsymbol{\Phi}+\beta \dot{\boldsymbol{\Phi}})=\mathbf{Q}^{e}(\mathbf{q}, \mathbf{u})
$$

The Lagrange multipliers vector $\lambda$ is hence de facto approximated by $\alpha \mathbf{\Phi}+\beta \dot{\boldsymbol{\Phi}}$. Higher values of the penalty factors imply better approximations, but also additional numerical ill-conditioning in the resolution of the equations. Depending on the application, a choice of the penalty factors has then to be made in order to find a balance between accuracy and computational efficiency and this represents a disadvantage of this approach of dealing with the constraints.

On the other hand, this choice offers a few decisive advantages. First, the Lagrangian multipliers vector $\lambda$ is not anymore part of the equations and the only unknown variable is the generalized coordinates vector $\mathbf{q}$ which can be found by solving the system of Ordinary Differential Equations (ODEs) of Eq. 5. This simplifies the estimation problem which remains unconstrained. Furthermore it also offers advantages with respect to the alternative approach that would lead to a set of ODEs, i.e. writing the EOMs in terms of the set of the only independent coordinates. Indeed, by virtue of the constraint equations only a subset of $n_{D O F}=n_{G C}-n_{C}$ of the generalized coordinates in vector $\mathbf{q}$ is independent, while the others can be written as a function of the independent ones. Hence Eq. 4 could be rewritten as an ODE in terms of only such independent generalized coordinates. However in this approach the additional steps of expressing dependent coordinates in function of independent ones and of managing the possible change of set of independent coordinates are required [21], which might become a hurdle during estimation and are not required when a penalty formulation is adopted.

Different formulations of the EOMs would then lead to different estimation problem definitions and resolutions, but a more comprehensive and detailed comparison of all the different possibilities is out of the scope of this work.

In order to more explicitly write the terms in Eq. 5 in function of the generalized coordinates vector and its time derivatives, a particular choice of the generalized coordinates has to be made. In this work so-called flexible natural coordinates are used [32], but a general notation is kept in order to derive a procedure that can be used for any choice of set of generalized coordinates. It is only added here, that as in many other generalized coordinates choice in flexible multibody dynamics, $\mathbf{q}$ is composed by stacking the vectors of generalized coordinates $\mathbf{q}^{i}$ of each body $i$. Each of the vectors $\mathbf{q}^{i}$ is then composed as $\mathbf{q}^{i}=\left[\mathbf{q}_{r}^{i}, \mathbf{q}_{f}^{i}\right]^{T}$ where $\mathbf{q}_{r}^{i}$ contains the rigid coordinates used to describe the global rigid motion of each body, while $\mathbf{q}_{f}^{i}$ contains the flexible coordinates used to describe the part of the motion related to each body's flexibility. This distinction is useful in Section 3 to describe the modeling of the flexible bodies.

A quite general form of the EOMs of such a type of mechanical system can thus be written as

$$
\mathbf{M}(\mathbf{q}) \ddot{\mathbf{q}}+\mathbf{f}_{n l}(\mathbf{q}, \dot{\mathbf{q}}, \mathbf{u})=\mathbf{0}
$$


where $\mathbf{M}$ is the mass matrix and $\mathbf{f}_{n l}$ is a generic non-linear term which groups all the remaining terms. For particular choices of the generalized coordinates set, the mass matrix $\mathbf{M}$ is not invertible, as for instance in [31] and [32], and for this reason it is not possible to retrieve an explicit state-space description of the form of Eq. 2. A more general description that does not assume the existence of the inverse of the mass matrix $\mathbf{M}^{-1}$ is then aimed herewith. By introducing the vector of generalized velocities $\mathbf{v}$ and by writing the relationship between the latter and the generalized coordinate vector $\mathbf{q}$ along with Eq. 6 , the following system of equations is obtained

$$
\left\{\begin{array}{l}
\mathbf{g}_{1}(\dot{\mathbf{q}}, \dot{\mathbf{v}}, \mathbf{q}, \mathbf{v}, \mathbf{u})=\mathbf{v}-\dot{\mathbf{q}}=\mathbf{0} \\
\mathbf{g}_{2}(\dot{\mathbf{q}}, \dot{\mathbf{v}}, \mathbf{q}, \mathbf{v}, \mathbf{u})=\mathbf{M}(\mathbf{q}) \dot{\mathbf{v}}+\mathbf{f}_{n l}(\mathbf{q}, \mathbf{v}, \mathbf{u})=\mathbf{0}
\end{array}\right.
$$

where the real-valued functions $\mathbf{g}_{1}$ and $\mathbf{g}_{2}$ have been defined for later convenience. The system in Eq. 7 fits in the implicit state-space description of Eq. 1 when the state vector is defined as

$$
\mathbf{x}=\left[\begin{array}{l}
\mathbf{q} \\
\mathbf{v}
\end{array}\right]
$$

\subsection{Time-discretization through an implicit scheme}

In this work a discrete-time EKF is used in order to estimate the states of the system. For this reason it is first needed to time-discretize Eq. 1. As the system is modeled as a flexible multibody model, an implicit timediscretization scheme is selected to cope with the numerical stiffness of Eq. 6 and to allow having a stable solution with a sufficiently large time step size. Backward Euler is used in this work, for which the following integration rules can be written

$$
\left\{\begin{array}{l}
\mathbf{v}_{k+1}=\frac{1}{h}\left(\mathbf{q}_{k+1}-\mathbf{q}_{k}\right) \\
\dot{\mathbf{v}}_{k+1}=\frac{1}{h}\left(\mathbf{v}_{k+1}-\mathbf{v}_{k}\right)
\end{array}\right.
$$

where $h$ represents the time step size, while $k$ and $k+1$ represent the current and next time step respectively. By plugging the integration rules of Eq. 9 into the EOMs written for time step $k+1$ which read

$$
\mathbf{M}\left(\mathbf{q}_{k+1}\right) \dot{\mathbf{v}}_{k+1}+\mathbf{f}_{n l}\left(\mathbf{q}_{k+1}, \mathbf{v}_{k+1}, \mathbf{u}_{k+1}\right)=\mathbf{0},
$$

the discrete-time EOMs can be found, for which a formal expression can be written as

$$
\mathbf{g}_{2 d}\left(\mathbf{q}_{k+1}, \mathbf{q}_{k}, \mathbf{v}_{k}, \mathbf{u}_{k+1}\right)=\mathbf{0}
$$

in which the dependency on $\mathbf{v}_{k+1}$ is resolved using the first integration rule of Eq. 9. If the generalized coordinates $\mathbf{q}_{k}$ and generalized velocities $\mathbf{v}_{k}$ at time step $k$ and the input vector $\mathbf{u}_{k+1}$ at time step $k+1$ are given, Eq. 11 allows to find the generalized coordinates $\mathbf{q}_{k+1}$ at time step $k+1$. The integration rules can then be used to find the generalized velocities $\mathbf{v}_{k+1}$ and time derivatives of the generalized velocities $\dot{\mathbf{v}}_{k+1}$ at time step $k+1$. In order to solve the non-linear Eq. 11 for $\mathbf{q}_{k+1}$, a Newton-Raphson iterative scheme can be applied with relative iteration matrix given by

$$
\mathbf{J}_{N R}=-\left[\mathbf{K}_{t}+\beta^{\prime} \mathbf{C}_{t}+\gamma^{\prime} \mathbf{M}_{t}\right]^{-1}
$$

where $\mathbf{K}_{t}, \mathbf{C}_{t}$ and $\mathbf{M}_{t}$ are the stiffness, damping and mass tangent matrices defined as

$$
\mathbf{K}_{t}=\frac{\partial \mathbf{g}_{2}}{\partial \mathbf{q}}, \quad \mathbf{C}_{t}=\frac{\partial \mathbf{g}_{2}}{\partial \mathbf{v}}, \quad \mathbf{M}_{t}=\frac{\partial \mathbf{g}_{2}}{\partial \dot{\mathbf{v}}}
$$

while the coefficients matrices $\beta^{\prime}$ and $\gamma^{\prime}$ are determined from the integration rules in Eq. 9 as

$$
\beta^{\prime}=\frac{\partial \mathbf{v}_{k+1}}{\partial \mathbf{q}_{k+1}}=\frac{1}{h} \mathbf{I}, \quad \gamma^{\prime}=\frac{\partial \dot{\mathbf{v}}_{k+1}}{\partial \mathbf{q}_{k+1}}=\frac{1}{h^{2}} \mathbf{I}
$$

The relation between the vectors $\mathbf{q}_{k+1}, \mathbf{v}_{k+1}$ and $\dot{\mathbf{v}}_{k+1}$ imposed by the integration rules in Eq. 9 and the chain rule have been considered in order to derive $\mathbf{J}_{N R}$ from Eq. 10. Furthermore only the tangent matrices related to the continuoustime EOMs are employed as visible in Eq. 13 and the residual vector at each iteration can be found by using the 
left hand side of Eq. 10. As a result, Eq. 11 and the tangent matrices associated to it do not need to be explicitly determined.

Note also that in the cases in which $\mathbf{M}$ is not invertible, $\mathbf{J}_{N R}$ still exists thanks to the contributions related to the tangent stiffness and tangent damping matrices $\mathbf{K}_{t}$ and $\mathbf{C}_{t}$.

The time-discretization of the first of Eqs. 7 is then performed by simply considering the first of the integration rules of Eq. 9 , i.e. by writing

$$
\mathbf{g}_{1 d}\left(\mathbf{q}_{k+1}, \mathbf{v}_{k+1}, \mathbf{q}_{k}, \mathbf{v}_{k}, \mathbf{u}_{k+1}\right)=\mathbf{v}_{k+1}-\frac{1}{h}\left(\mathbf{q}_{k+1}-\mathbf{q}_{k}\right)=\mathbf{0}
$$

Eq. 15 and Eq. 11 can be formally combined in order to compactly describe the discrete-time form of Eq. 1, i.e.

$$
\mathbf{g}_{d}\left(\mathbf{x}_{k+1}, \mathbf{x}_{k}, \mathbf{u}_{k+1}\right)=\mathbf{0}
$$

Finally note that different implicit time-discretization schemes can be adopted without altering the procedure, by simply changing the integration rules in Eq. 9 and adapting the coefficient matrices of Eq. 14 .

\subsection{Linearization of the state equation}

As a discrete-time EKF is used in this work in order to estimate the states of the system, linearizations of the system around each states estimate are needed. More in detail the so-called system Jacobian matrices are needed which are defined as follows

$$
\mathbf{A}=\frac{\partial \mathbf{f}_{d}}{\partial \mathbf{x}_{k}}, \quad \mathbf{B}=\frac{\partial \mathbf{f}_{d}}{\partial \mathbf{u}_{k+1}},
$$

where $\mathbf{f}_{d}$ is the underlying explicit function associated with $\mathbf{g}_{d}$ and which relates $\mathbf{x}_{k}$ and $\mathbf{u}_{k+1}$ to $\mathbf{x}_{k+1}$ i.e.

$$
\mathbf{x}_{k+1}=\mathbf{f}_{d}\left(\mathbf{x}_{k}, \mathbf{u}_{k+1}\right)
$$

The Jacobian matrices related to such explicit function $\mathbf{f}_{d}$ are needed as the discrete-time EKF in its traditional form has been formulated for explicit dynamic systems of the form of Eq. 2, as reported e.g. in [17]. Function $\mathbf{g}_{d}$ of Eq. 16 is assumed to be continuously differentiable in all its variables and consequently the theorem of the implicit function ensures that the explicit function $\mathbf{f}_{d}$ exists locally. Then, the Taylor first order expansion of $\mathbf{g}_{d}$ can be written as

$$
\mathbf{g}_{d}\left(\mathbf{x}_{k+1}, \mathbf{x}_{k}, \mathbf{u}_{k+1}\right)=\mathbf{g}_{d}^{0}+\left.\frac{\partial \mathbf{g}_{d}}{\partial \mathbf{x}_{k+1}}\right|_{0}\left(\mathbf{x}_{k+1}-\mathbf{x}_{k+1}^{0}\right)+\left.\frac{\partial \mathbf{g}_{d}}{\partial \mathbf{x}_{k}}\right|_{0}\left(\mathbf{x}_{k}-\mathbf{x}_{k}^{0}\right)+\left.\frac{\partial \mathbf{g}_{d}}{\partial \mathbf{u}_{k+1}}\right|_{0}\left(\mathbf{u}_{k+1}-\mathbf{u}_{k+1}^{0}\right)+\ldots
$$

where $\left(\mathbf{x}_{k+1}^{0}, \mathbf{x}_{k}^{0}, \mathbf{u}_{k+1}^{0}\right)$ represents the linearization point and $\mathbf{g}_{d}\left(\mathbf{x}_{k+1}^{0}, \mathbf{x}_{k}^{0}, \mathbf{u}_{k+1}^{0}\right)$ has been indicated by $\mathbf{g}_{d}^{0}$ for convenience of notation. If such expansion is plugged into Eq. 16 and $\mathbf{x}_{k+1}$ is made explicit, the following equation can be obtained

$$
\mathbf{x}_{k+1}=\mathbf{x}_{k+1}^{0}-\left[\left.\frac{\partial \mathbf{g}_{d}}{\partial \mathbf{x}_{k+1}}\right|_{0}\right]^{-1}\left(\left.\frac{\partial \mathbf{g}_{d}}{\partial \mathbf{x}_{k}}\right|_{0}\left(\mathbf{x}_{k}-\mathbf{x}_{k}^{0}\right)+\left.\frac{\partial \mathbf{g}_{d}}{\partial \mathbf{u}_{k+1}}\right|_{0}\left(\mathbf{u}_{k+1}-\mathbf{u}_{k+1}^{0}\right)+\mathbf{g}_{d}^{0}\right)
$$

which holds when terms of order higher than one are neglected. By observing Eq. 20 is appears clear that the latter represents a local linearization of the explicit function $\mathbf{f}_{d}$, from which it follows that the Jacobian matrices $\mathbf{A}_{0}$ and $\mathbf{B}_{0}$ related to the linearization point $\left(\mathbf{x}_{k+1}^{0}, \mathbf{x}_{k}^{0}, \mathbf{u}_{k+1}^{0}\right)$ can be computed as

$$
\mathbf{A}_{0}=-\left.\left[\left.\frac{\partial \mathbf{g}_{d}}{\partial \mathbf{x}_{k+1}}\right|_{0}\right]^{-1} \frac{\partial \mathbf{g}_{d}}{\partial \mathbf{x}_{k}}\right|_{0}=-\left.\left.\mathbf{G}_{\mathbf{x}_{k+1}}^{-1}\right|_{0} \mathbf{G}_{\mathbf{x}_{k}}\right|_{0}, \quad \mathbf{B}_{0}=-\left.\left[\left.\frac{\partial \mathbf{g}_{d}}{\partial \mathbf{x}_{k+1}}\right|_{0}\right]^{-1} \frac{\partial \mathbf{g}_{d}}{\partial \mathbf{u}_{k+1}}\right|_{0}=-\left.\left.\mathbf{G}_{\mathbf{x}_{k+1}}^{-1}\right|_{0} \mathbf{G}_{\mathbf{u}_{k+1}}\right|_{0}
$$

where the matrices of derivatives $\mathbf{G}_{\mathbf{x}_{k+1}}, \mathbf{G}_{\mathbf{x}_{k}}$ and $\mathbf{G}_{\mathbf{u}_{k+1}}$ have been introduced. Note that it has to be assumed that the matrix $\mathbf{G}_{\mathbf{x}_{k+1}}$ is invertible in order to perform the linearization. Matrices $\mathbf{G}_{\mathbf{x}_{k+1}}$ and $\mathbf{G}_{\mathbf{x}_{k}}$ can be more explicitly determined by considering the structure of the state vector $\mathbf{x}$ as defined by Eq. 8, in addition to considering Eq. 15, Eq. 10 and the satisfaction of the integration rules of Eq. 9. The following expressions can hence be found

$$
\begin{aligned}
\mathbf{G}_{\mathbf{x}_{k+1}} & =\left[\begin{array}{cc}
\frac{\partial \mathbf{g}_{1 d}}{\partial \mathbf{q}_{k+1}} & \frac{\partial \mathbf{g}_{1 d}}{\partial \mathbf{v}_{k+1}} \\
\frac{\partial \mathbf{g}_{2 d}}{\partial \mathbf{q}_{k+1}} & \frac{\partial \mathbf{g}_{2 d}}{\partial \mathbf{v}_{k+1}}
\end{array}\right]=\left[\begin{array}{cc}
-\frac{1}{h} \mathbf{I} & \mathbf{I} \\
\mathbf{K}_{t}+\beta^{\prime} \mathbf{C}_{t}+\gamma^{\prime} \mathbf{M}_{t} & \mathbf{0}
\end{array}\right], \\
\mathbf{G}_{\mathbf{x}_{k}} & =\left[\begin{array}{ll}
\frac{\partial \mathbf{g}_{1 d}}{\partial \mathbf{q}_{k}} & \frac{\partial \mathbf{g}_{1 d}}{\partial \mathbf{v}_{k}} \\
\frac{\partial \mathbf{g}_{2 d}}{\partial \mathbf{q}_{k}} & \frac{\partial \mathbf{g}_{2 d}}{\partial \mathbf{v}_{k}}
\end{array}\right]=\left[\begin{array}{cc}
\frac{1}{h} \mathbf{I} & \mathbf{0} \\
\phi^{\prime} \mathbf{C}_{t}+\chi^{\prime} \mathbf{M}_{t} & \psi^{\prime} \mathbf{M}_{t}
\end{array}\right]
\end{aligned}
$$


where the chain rule has been used to compute the derivatives of $\mathbf{g}_{2 d}$ and the new coefficient matrices $\phi^{\prime}, \chi^{\prime}$ and $\psi^{\prime}$ are again determined from the integration rules in Eq. 9 as

$$
\phi^{\prime}=\psi^{\prime}=\frac{\partial \mathbf{v}_{k+1}}{\partial \mathbf{q}_{k}}=-\frac{1}{h} \mathbf{I}, \quad \chi^{\prime}=\frac{\partial \dot{\mathbf{v}}_{k+1}}{\partial \mathbf{q}_{k}}=-\frac{1}{h^{2}} \mathbf{I}
$$

Eq. 22 allows to analytically compute the linearization matrices in function of the tangent matrices of the continuoustime EOMs and of the coefficient matrices of Eq. 14 and of Eq. 23. This provides the means for a computationally efficient linearization procedure given that the tangent matrices $\mathbf{K}_{t}, \mathbf{C}_{t}$ and $\mathbf{M}_{t}$ are analytically available.

The reader should again note that in the cases in which $\mathbf{M}$ is not invertible, the existence of the inverse of $\mathbf{G}_{\mathbf{x}_{k+1}}$ has to be guaranteed by the contributions related to the tangent stiffness and tangent damping matrices $\mathbf{K}_{t}$ and $\mathbf{C}_{t}$. Furthermore, a different time-discretization scheme can be used by simply adapting the coefficient matrices of Eq. 23, in addition to the integration rules of Eq. 9 and to the coefficient matrices of Eq. 14.

For what concerns instead with $\mathbf{G}_{\mathbf{u}_{k+1}}$ the following holds

$$
\mathbf{G}_{\mathbf{u}_{k+1}}=\left[\begin{array}{c}
\mathbf{0} \\
\frac{\partial \mathbf{g}_{2 d}}{\partial \mathbf{u}_{k+1}}
\end{array}\right]
$$

in which the partial derivatives with respect to $\mathbf{u}_{k+1}$ might or might not be available analytically. In this work such derivatives are computed by numerical differentiation, but this does not represent a hurdle from a computational point of view as the number of inputs is generally limited.

Besides the system governing equation discussed so far, i.e. Eq. 1 for the continuous-time version or Eq. 16 for the discrete-time counterpart, it is important to introduce the so-called measurement equation. The latter relates the system state vector $\mathbf{x}$ to the measurement vector $\mathbf{y} \in \mathbb{R}^{n_{O}}$ as follows

$$
\mathbf{y}=\mathbf{h}(\mathbf{x})
$$

where $\mathbf{h}$ is a real-valued function which has to be properly defined depending on the measurement quantities contained in vector $\mathbf{y}$ and further details about this are given in Appendix A. A linearization of such equation is also needed, which reads

$$
\mathbf{y}=\mathbf{h}\left(\mathbf{x}^{0}\right)+\left.\frac{\partial \mathbf{h}}{\partial \mathbf{x}}\right|_{0}\left(\mathbf{x}-\mathbf{x}^{0}\right)=\mathbf{h}\left(\mathbf{x}^{0}\right)+\mathbf{H}_{0}\left(\mathbf{x}-\mathbf{x}^{0}\right)
$$

where the new Jacobian matrix $\mathbf{H}_{0}$ has been introduced.

\subsection{Estimation procedure}

The governing equation of Eq. 16 and the measurement equation of Eq. 25 discussed so far are purely deterministic. In optimal estimation a stochastic component is normally also introduced in order to describe model uncertainties. By introducing such source of uncertainties, the full discrete-time state-space description of the dynamic system can be written as follows

$$
\left\{\begin{array}{l}
\mathbf{g}_{d}\left(\mathbf{x}_{k+1}, \mathbf{x}_{k}, \mathbf{u}_{k+1}\right)=\mathbf{w}_{k} \\
\mathbf{y}_{k+1}=\mathbf{h}\left(\mathbf{x}_{k+1}\right)+\mathbf{v}_{k+1}
\end{array}\right.
$$

where the so-called process noise vector and measurement noise vector, $\mathbf{w}_{k}$ and $\mathbf{v}_{k}$ respectively, have been introduced. It is assumed that both $\mathbf{w}_{k}$ and $\mathbf{v}_{k}$ are white, zero-mean, uncorrelated random processes with known covariance matrices $\mathbf{Q}_{k}$ and $\mathbf{R}_{k}$ respectively. As it can be seen from Eq. 27 both the process noise and the measurement noise are considered to be additive in this work, but the procedure described can easily be adapted if a more general noise type is to be used.

As the aim of this work is to estimate both states and unknown inputs of the system, a distinction is made between the vector of the known inputs $\mathbf{u}^{K}$ and the vector of the unknown inputs $\mathbf{u}^{U K}$, which together make up the full input vector $\mathbf{u}$ as

$$
\mathbf{u}=\left[\begin{array}{c}
\mathbf{u}^{K} \\
\mathbf{u}^{U K}
\end{array}\right]
$$


The following so-called random walk model is then introduced for the vector of unknown inputs $\mathbf{u}^{U K}$

$$
\mathbf{u}_{k+1}^{U K}=\mathbf{u}_{k}^{U K}+\mathbf{w}_{k}^{U K}
$$

in which $\mathbf{w}_{k}^{U K}$ is assumed to be a white, zero-mean, uncorrelated random processes with known covariance matrix $\mathbf{Q}_{k}^{U K}$. The discrete-time state-representation of the so-classed augmented system can then be obtained by combining Eq. 27 and Eq. 29, i.e.

$$
\left\{\begin{array}{l}
\mathbf{g}_{d}\left(\mathbf{x}_{k+1}, \mathbf{x}_{k}, \mathbf{u}_{k+1}\right)=\mathbf{w}_{k} \\
\mathbf{u}_{k+1}^{U K}=\mathbf{u}_{k}^{U K}+\mathbf{w}_{k}^{U K} \\
\mathbf{y}_{k+1}=\mathbf{h}\left(\mathbf{x}_{k+1}\right)+\mathbf{v}_{k+1}
\end{array}\right.
$$

for which a new augmented state vector as well as a new augmented process noise covariance matrix are introduced as

$$
\mathbf{x}^{a}=\left[\begin{array}{c}
\mathbf{x} \\
\mathbf{u}^{U K}
\end{array}\right], \quad \mathbf{Q}_{k}^{a}=\left[\begin{array}{cc}
\mathbf{Q}_{k} & \mathbf{0} \\
\mathbf{0} & \mathbf{Q}_{k}^{U K}
\end{array}\right]
$$

and for which the augmented system Jacobian matrices are defined as

$$
\mathbf{A}^{a}=\frac{\partial \mathbf{f}_{d}^{a}}{\partial \mathbf{x}^{a}}=\left[\begin{array}{cc}
\mathbf{A} & \mathbf{B}^{U K} \\
\mathbf{0} & \mathbf{I}
\end{array}\right], \quad \mathbf{B}^{a}=\frac{\partial \mathbf{f}_{d}^{a}}{\partial \mathbf{u}^{K}}=\left[\begin{array}{c}
\mathbf{B}^{K} \\
\mathbf{0}
\end{array}\right]
$$

where $\mathbf{f}_{d}^{a}$ is the augmented explicit function associated with Eq. 16 and Eq. 29 together, while $\mathbf{B}^{U K}$ and $\mathbf{B}^{K}$ are the matrices obtained from matrix $\mathbf{B}$ by keeping only the columns related to the unknown and known inputs respectively. Furthermore, the augmented measurement equation Jacobian matrix is introduced as

$$
\mathbf{H}^{a}=\frac{\partial \mathbf{h}}{\partial \mathbf{x}^{a}}
$$

It appears clear from Eq. 27 and from Eq. 30 that both the state vector $\mathbf{x}$ and the augmented state vector $\mathbf{x}^{a}$ are now modeled as random processes.

The aim of the estimation is to provide the estimate of the first two statistical moments of the augmented state vector $\mathbf{x}^{a}$ given the sequence of measurements $\left\{\mathbf{y}_{k}\right\}$ and known inputs $\left\{\mathbf{u}_{k}^{K}\right\}$. The first moment corresponds to the mean value and the estimate of such quantity for the augmented state vector, the state vector and the unknown input vector is indicated with $\hat{\mathbf{x}}^{a}, \hat{\mathbf{x}}$ and $\hat{\mathbf{u}}^{U K}$ respectively. The second moment corresponds instead to the augmented state error covariance matrix $\mathbf{P}^{a}$ defined as

$$
\mathbf{P}_{k}^{a}=\mathbf{E}\left[\left(\mathbf{x}_{k}^{a}-\hat{\mathbf{x}}_{k}^{a}\right)\left(\mathbf{x}_{k}^{a}-\hat{\mathbf{x}}_{k}^{a}\right)^{T}\right]
$$

where $\mathbf{E}$ represents the expected value operator. Furthermore in the following the superscript $\square^{-}$is used to indicate quantities that are estimated by making use of measurements up to time step $k$, i.e. to indicate so-called $a$-priori estimates. The superscript $\square^{+}$is instead used to indicate quantities that are estimated by making use of measurements up to time step $k+1$, i.e. to indicate so-called a-posteriori estimates.

It is now possible to illustrate the full estimation procedure which is obtained by applying a linear KF to the linearized version of system of Eq. 30. Note that the second equation in such system is already linear, while linearized versions of the deterministic parts of the other two are given in Eq. 20 and in Eq. 26. The estimation is recursive and proceeds for each time step as indicated in the following table: 
Compute the a-priori estimate of the unknown input vector mean as

$$
\hat{\mathbf{u}}_{k+1}^{U K-}=\hat{\mathbf{u}}_{k}^{U K+}
$$

Compose the input vector as

$$
\mathbf{u}_{k+1}=\left[\begin{array}{c}
\mathbf{u}_{k+1}^{K} \\
\hat{\mathbf{u}}_{k+1}^{U K-}
\end{array}\right]
$$

Compute the a-priori estimate of the state vector mean by solving

$$
\mathbf{g}_{d}\left(\hat{\mathbf{x}}_{k+1}^{-}, \hat{\mathbf{x}}_{k}^{+}, \mathbf{u}_{k+1}\right)=\mathbf{0}
$$

as explained in sub-section (2.2).

Propagate the augmented state error covariance matrix by means of

$$
\mathbf{P}_{k+1}^{a-}=\mathbf{A}_{k}^{a} \mathbf{P}_{k}^{a+} \mathbf{A}_{k}^{a T}+\mathbf{Q}_{k}^{a}
$$

Compute the Kalman gain as

$$
\mathbf{K}_{k+1}=\mathbf{P}_{k+1}^{a-} \mathbf{H}_{k}^{a T}\left(\mathbf{H}_{k}^{a} \mathbf{P}_{k+1}^{a-} \mathbf{H}_{k}^{a T}+\mathbf{R}_{k+1}\right)^{-1}
$$

Compute the a-posteriori estimate of the state vector and of the unknown input vector as

$$
\hat{\mathbf{x}}_{k+1}^{a+}=\left[\begin{array}{c}
\hat{\mathbf{x}}_{k+1}^{+} \\
\hat{\mathbf{u}}_{k+1}^{U K+}
\end{array}\right]=\left[\begin{array}{c}
\hat{\mathbf{x}}_{k+1}^{-} \\
\hat{\mathbf{u}}_{k+1}^{U K-}
\end{array}\right]+\mathbf{K}_{k+1}\left(\mathbf{y}_{k+1}-\mathbf{h}\left(\hat{\mathbf{x}}_{k+1}^{-}\right)\right)
$$

Compute the a-posteriori augmented state error covariance matrix as

$$
\mathbf{P}_{k+1}^{a+}=\left(\mathbf{I}-\mathbf{K}_{k+1} \mathbf{H}_{k}^{a}\right) \mathbf{P}_{k+1}^{a-}
$$

Note that the input vector $\mathbf{u}_{k+1}$ is only a particular case of parameter in Eq. 16. The exposed procedure can hence be easily extended to the case of estimation of additional system parameters.

Furthermore, by carrying out only the steps in Eqs. from 37 to 41 for each time step and by replacing the augmented matrices $\mathbf{A}_{k}^{a}, \mathbf{P}_{k}^{a}, \mathbf{Q}_{k}^{a}, \mathbf{H}_{k}^{a}$ and $\mathbf{R}_{k}^{a}$ with their non-augmented counterparts $\mathbf{A}_{k}, \mathbf{P}_{k}, \mathbf{Q}_{k}, \mathbf{H}_{k}$ and $\mathbf{R}_{k}$, the estimation of only states can be performed in case all the inputs are known.

\section{The McPherson suspension test rig}

The estimation procedure presented in section 2 has been validated on the suspension test rig shown in Figure 1. The latter is based on a McPherson suspension system which is attached to a fixed frame at the regular connections-tobody locations and loaded at the tire contact patch by a six DOF hydraulic shaker capable of reproducing operational road loads. This is close to the envisioned application case of RLDA for automotive durability testing.

Figure 2 shows a more detailed view of the McPherson suspension architecture and reports an illustration of the employed system model. As shown in Figure 2a the suspension includes a control arm, a lower strut, an upper strut and a tierod all connected to a steering knuckle. The four connection-to-body locations are indicated with red dots.

The system is modeled as a flexible multibody model as illustrated in Figure $2 b$. The control arm and the steering knuckle are considered to be flexible bodies, while the tierod, the lower strut and the upper strut are described as rigid bodies. The frame is considered to be rigid and fixed to the ground. Spherical joints connect the control arm, the tierod and the upper strut to the frame. The steering knuckle is then connected to the lower strut by a rigid joint and to both the tierod and the control arm by two additional spherical joints. Finally, the lower strut and upper strut are connected to each other by a cylindrical joint.

The system has hence one global degree of freedom corresponding to the global vertical motion, in addition to the flexible degrees of freedom related to the two flexible components. 


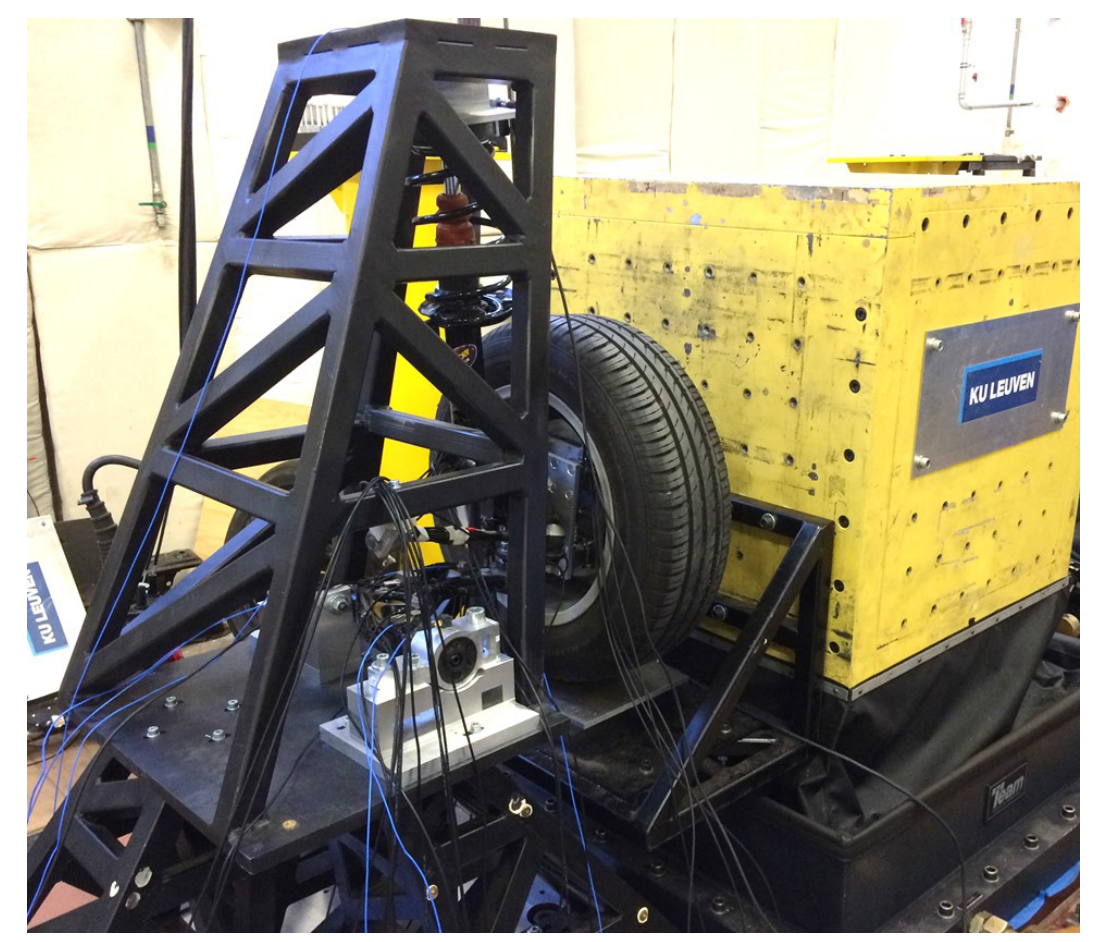

Figure 1: The validation test rig is based on a McPherson suspension attached to a frame on one side and loaded by a six DOF hydraulic shaker capable of reproducing road loads on the other side.

It is assumed that the two flexible bodies, i.e. the steering knuckle and the control arm, undergo only small deformations and are modeled as reduced order linear finite element models. The steering knuckle is mainly composed of quadratic tetrahedron elements while the control arm is mainly composed of bi-linear quadrilateral shell elements with a minority of linear triangular shell elements [36]. Rigid elements are then used at the connections to the adjacent bodies. Such rigid elements connect all the nodes close to the connection to central independent nodes, as visible in Figure $2 \mathrm{~b}$. The independent nodes are then used for the multibody joints definitions. The reduction of each finite element model is performed by the modal truncation augmentation method as explained in [37]. The reduction basis $\Psi^{i}$ used for body $i$ is composed as $\Psi^{i}=\left[\Psi_{c o n}^{i} \Psi_{n m}^{i}\right]$, where $\boldsymbol{\Psi}_{c o n}^{i}$ contains all the constraint modes [38] related to the interface of body $i$ while $\Psi_{n m}^{i}$ contains some of the normal modes of each body $i$. The inclusion of the constrain modes ensures that the reduced model is as accurate as the full model with respect to static loads exchanged through the connections, hence ensuring representative models at static regime. The static and quasi-static regime of deformation is indeed the most relevant for the application considering that excitation signals only up to $40 \mathrm{~Hz}$ have been used as later on explained in this section. The first elastic normal mode at $242 \mathrm{~Hz}$ is then included in the control arm normal modes set while no additional normal mode is used for the normal modes set of the steering knuckle which results to be a pretty stiff component with first eigen-frequency at $951 \mathrm{~Hz}$. The flexible generalized coordinates vector $\mathbf{q}_{f}^{i}$ of each body $i$ hence contains the modal participation factors related to each vector of the reduction basis represented by $\Psi$.

A linear spring force element is used to model the suspension main spring, while a non linear friction force element is used to model the viscous friction as well as the contact friction between the lower and upper damper. The definition laws for such force elements are reported in Table 1 . In the table expressions, $F_{s}$ and $F_{f}$ represent the signed amplitude values of the spring element force and of the friction element force respectively. The latter are exchanged between the upper strut and the lower strut along the two force elements axes. $x_{r e l, s}$ and $v_{\text {rel, }}$ respectively represent instead the relative displacement and relative velocity between the upper and the lower strut along the two force elements axes. The relative parameters are also reported along with the adopted values which have been obtained by optimization procedures in order match the real system response in terms of global response over a set of relevant 

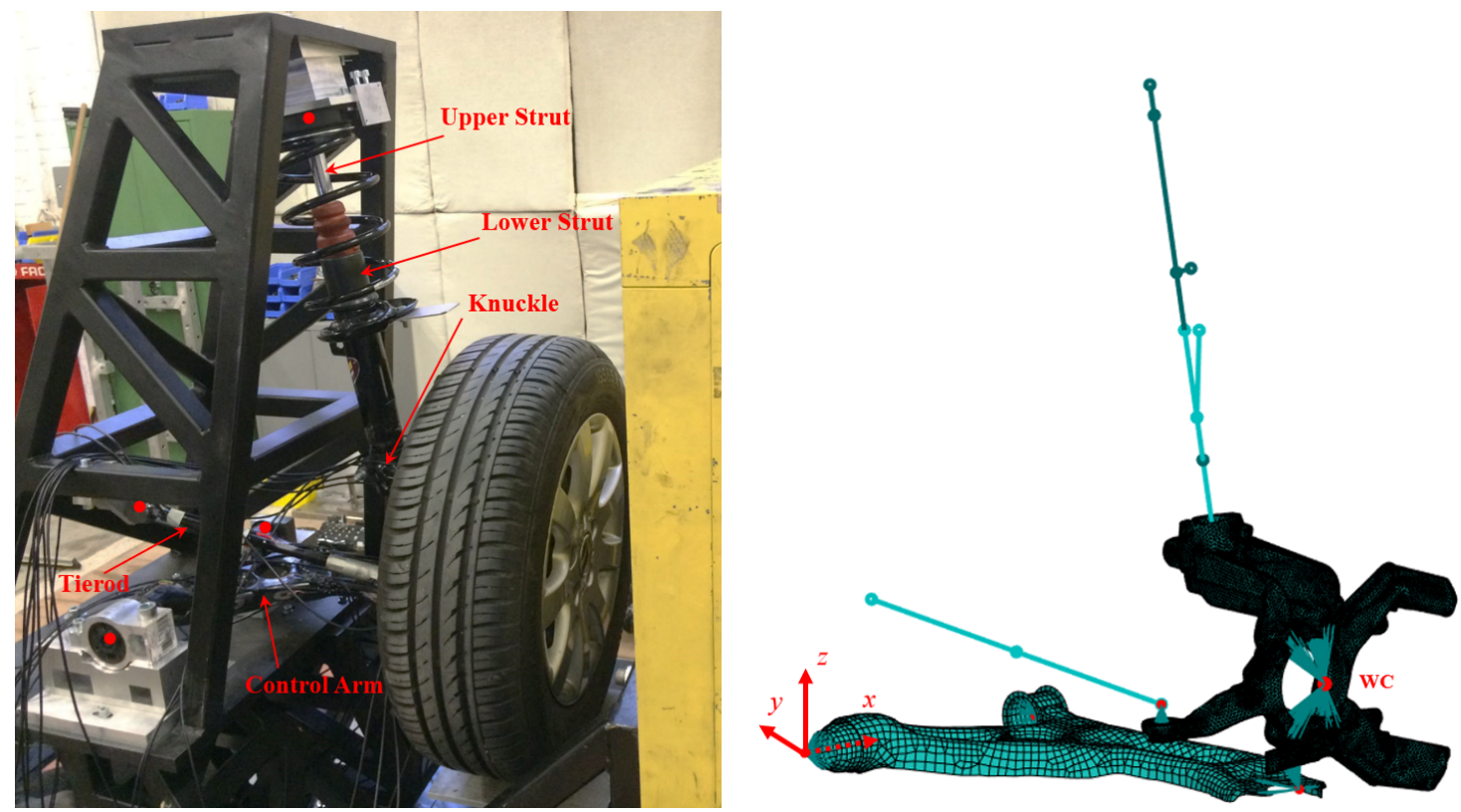

(a) The McPherson suspension is composed of a control (b) The control arm and the knuckle are modeled as flexiarm, a tierod, a lower strut and an upper strut all connected ble bodies, while the tierod, the upper strut and the lower to a steering knuckle. strut are modeled as rigid bodies. The external loads, i.e. the Wheel Center Loads, are applied to the Wheel Center (WC) which is a point rigidly connected to the knuckle.

Figure 2: The system has been modeled as a flexible multibody system.

experimental runs. The details of such model updating procedure are not reported herewith as they are not relevant for the remaining discussion.

\begin{tabular}{|c|c|c|}
\hline & Force element law & Relative parameters \\
\hline Spring & $F_{s}=K_{s} x_{r e l, s}$ & $K_{s}=23500 \mathrm{~N} / \mathrm{m}$ \\
\hline Friction & $\begin{array}{l}F_{f}=F_{v f}+F_{c f} \\
\left\{\begin{array}{l}F_{v f}=C_{1} v_{\text {rel }, f}, \quad \text { if } v_{\text {rel }, f}>0 \\
F_{v f}=C_{2} v_{\text {rel }, f}, \quad \text { if } v_{\text {rel }, f}<0\end{array}\right. \\
\begin{cases}F_{c f}=\frac{F_{a}}{v_{\text {trans }}} v_{\text {rel }, f}, \text { if }\left|v_{\text {rel }, f}\right|<v_{\text {trans }} \\
F_{c f}=F_{a}, \quad \text { if }\left|v_{\text {rel }, f}\right| \geqslant v_{\text {trans }}\end{cases} \end{array}$ & $\begin{aligned} C_{1} & =2207 \mathrm{~N} /(\mathrm{m} / \mathrm{s}) \\
C_{2} & =2280 \mathrm{~N} /(\mathrm{m} / \mathrm{s}) \\
F_{a} & =135 \mathrm{~N} \\
v_{\text {trans }} & =0.00883 \mathrm{~m} / \mathrm{s}\end{aligned}$ \\
\hline
\end{tabular}

Table 1: A linear spring element and a non-linear friction force element are used to model the internal forces exchanged between upper strut and lower strut. The parameters of the force laws of these force elements have been updated in order to match the real system global response for a number of relevant experimental runs.

The global reference frame of the model is centered in one of the connection-to-chassis locations of the control arm as reported in Figure $2 \mathrm{~b}$. The $x, y$ and $z$ axes correspond to the longitudinal, lateral and vertical directions of the vehicle respectively.

The external loads are considered to be applied at the Wheel Center (WC) which is a point rigidly attached to the knuckle and is also highlighted in Figure 2b. These external loads, which are indicated with $W C F x, W C F y, W C F z$, $W C M x, W C M y$ and WCMz, correspond to the WCLs for the suspension system and consist of three forces and three 
moments along and about the three global axes. The WC is located at the central location of the load cell shown in Figure 3a.

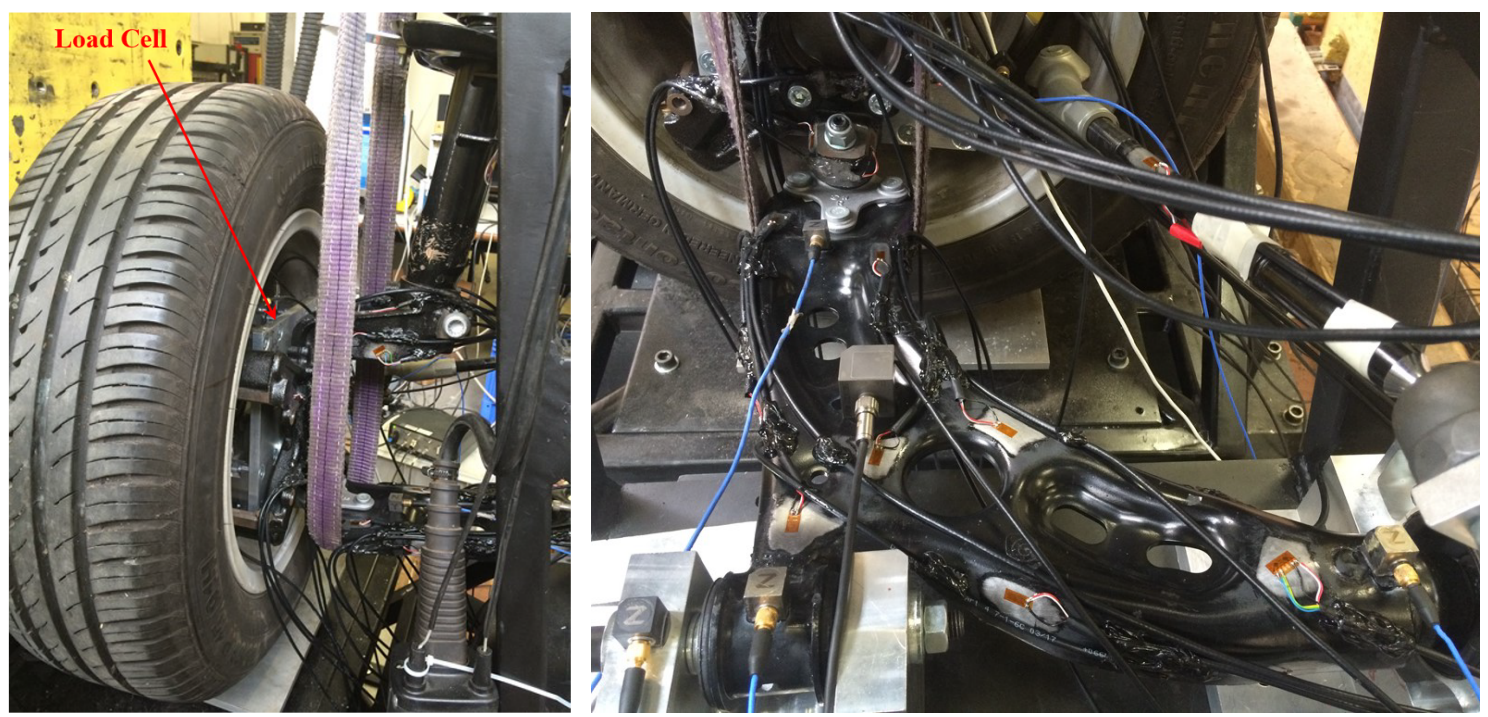

(a) A load cell is interposed between knuckle (b) Several strain gauges have been distributed over the steering knuckle and tire rim in order to measure the Wheel and the control arm. Some of them are used in the estimation procedure, Center Loads and provides the validation sig- while the others are used for validation. nals for such quantities.

Figure 3: The instrumentation includes sensors usually employed in Road Load Data Acquisition testing campaigns.

Such load cell is interposed between the knuckle and the tire rim hence it directly measures the WCLs providing the validation signals for such quantities. While being rigidly screwed on the knuckle side, the load cell includes bearings as well as a rotating spindle on the tire rim side such that the rolling motion of the tire is allowed.

Sensors have been distributed over knuckle and control arm for 34 strain gauges channels in total as shown in Figure $3 \mathrm{a}$ and Figure $3 \mathrm{~b}$. This represents the main part of the test rig instrumentation along with the load cell. Subsets of those sensor channels make up the sensor sets used in the estimation procedure as explained in Section 4, while the others are used as validation channels.

Additionally the signal from the internal displacement sensor of the hydraulic shaker which measures the vertical position of the latter has been acquired. This sensor signal has been used to define the experimental runs.

Table 2 lists the executed experimental runs that are relevant for this work. In all of them a purely vertical motion of the hydraulic shaker has been imposed. More in detail, sinusoidal motions of $5 \mathrm{~mm}$ of amplitude at $1 \mathrm{~Hz}, 4 \mathrm{~Hz}$ and $8 \mathrm{~Hz}$ have been imposed in the first three, while a road profile has been replicated in the last one. The time signal of the imposed road profile has been generated such that its Power Spectral Density (PSD) is representative of a road profile class 'A' according to the ISO standard [39] and assuming a constant vehicle speed of $30 \mathrm{~km} / \mathrm{h}$. A low-pass filter with cutoff frequency of $40 \mathrm{~Hz}$ has also been applied to such signal as a safety precaution with respect to the test rig integrity. The resulting time signal and PSD are shown in Figure 4. 


\begin{tabular}{cccc}
\hline ID & Signal type & Frequency $(\mathrm{Hz})$ & Amplitude $(\mathrm{mm})$ \\
\hline 1 & Sine & 1 & 5 \\
2 & Sine & 4 & 5 \\
3 & Sine & 8 & 5 \\
4 & Road A & $0-40$ & - \\
\hline
\end{tabular}

Table 2: The table shows the 4 experimental runs relevant for this work. In three of them sinusoidal motions of the hydraulic shaker have been imposed. A road profile has been instead reproduced in the fourth run.
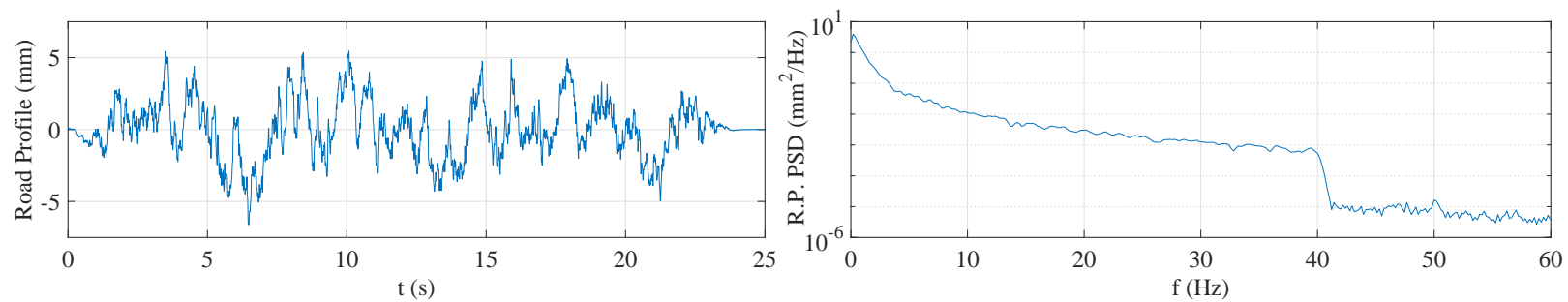

Figure 4: The reproduced road profile (see table 2) corresponds to a road class 'A' according to the ISO standard [39]. A constant vehicle speed of $30 \mathrm{~km} / \mathrm{h}$ has been assumed to retrieve the time signal reported. The related Power Spectral Density (PSD) is also reported.

\section{Sensors selection through an Optimal Sensor Placement strategy for non-linear systems}

The estimation approach proposed in section 2 is applied in section 5 to combine information from the flexible multibody model and subsets of the strain gauges described in section 3 . This work indeed envisions to jointly estimate states and mechanical inputs including the static behavior. Hence strain gauges are chosen to build the sensor sets, as they represent position level measurements that can ensure observability of inputs at $0 \mathrm{~Hz}$ as demonstrated in [9]. The fusion of information coming from different sensors types is out of scope in this work.

The next Subsection 4.1 explains the sensors selection procedure, in which some steps make use of WCLs measurements performed by means of the load cell. Comments about the use of the load cell are given in Subsection 4.2 .

\subsection{Sensor selection procedure}

The procedure outlined in Table 3 has been employed in order to define the sensors subsets to be used in the estimation process. It is based on the procedure proposed in [40] for linear structural systems, with the difference that the observability of the non-linear system of this work is treated as in [41].

Point 2 of this table ensures that the signal-to-noise ratios of the employed sensors channels are above a minimal threshold. Figure 5 shows the comparison of the Maximum Response Amplitude (MRA) at the different strains locations for the measured signals and for the ones obtained with forward simulation by using the measured WCLs as known inputs to the simulation model for the first 5 seconds of experimental run 2 of Table 2 . The MRA is defined as $\left(s_{\max }-s_{\min }\right) / 2$, where $s_{\max }$ and $s_{\min }$ are the maximum and minimum values of the considered signal. The sensors locations are ordered along the horizontal axis from the least responsive to the most responsive according to the measured values. The simulated MRA values corresponding to this experimental run have been used for the screening of point 2 while considering a threshold of $1 \mu \epsilon$. This latter value is only slightly higher than the standard deviation associated with the sensors noise which has values included between 0.2 and $0.3 \mu \epsilon$ depending on the specific measured channel. This implied the exclusion of the 5 strain gauges channels highlighted with red labels and corresponding to locations which are very weakly excited.

Point 3 of Table 3 is introduced in order to employ sensors with sufficient matching between simulation and experiments. This step has been carried out by visual inspection on the comparison between time series of simulated and 
Sensors selection procedure

1. Consider the initial pool of sensors composed of the 34 strain gauges channels distributed over Steering Knuckle and Control Arm

2. Remove sensors for which the amplitudes of the simulated responses of the model under specific training scenarios does not reach a certain threshold

3. Remove sensors which do not exhibit a sufficient level of matching between the simulated responses of the model and the corresponding measured responses for specific experimental runs

4. Keep the $M$ sensors that maximize a certain observability metric based on the Popov-BelovichHautus (PBH) observability criteria

Table 3: The sensors to be used in the estimation have been selected in order to maximize signal-to-noise ratios, simulated response matching with experiments and observability.

measured strains for experimental run 2 of Table 2. Figure 6 shows one example of a discarded sensor and one example of a retained sensor for this selection step, i.e. S9:+X and S14:+Y respectively. In this way the following sensors have been discarded: S17:+Y, S8+Y, S9:+X, S20:+Y, S24:+Y, S18:+Y, S25:+Y and S19:+Y.
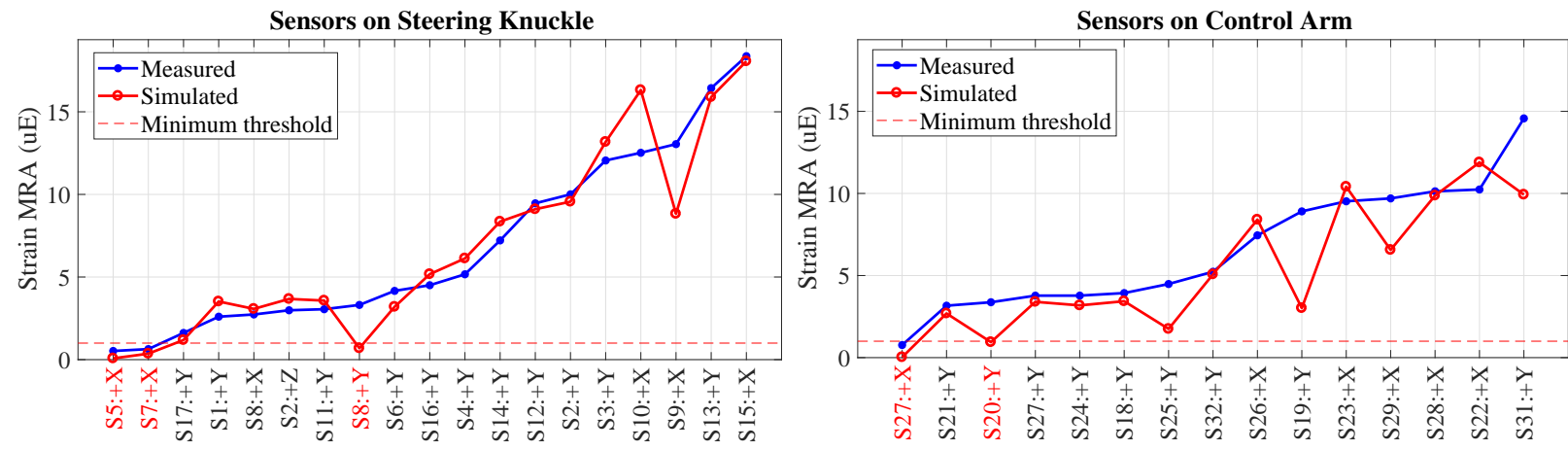

Figure 5: Comparison between measured strain Maximum Response Amplitude (MRA) and simulated strain MRA for experimental run 2 of Table 2. Sensors with simulated MRA lower than $1 \mu \epsilon$ are discarded by the sensor selection algorithm according to step 2 of Table 3.
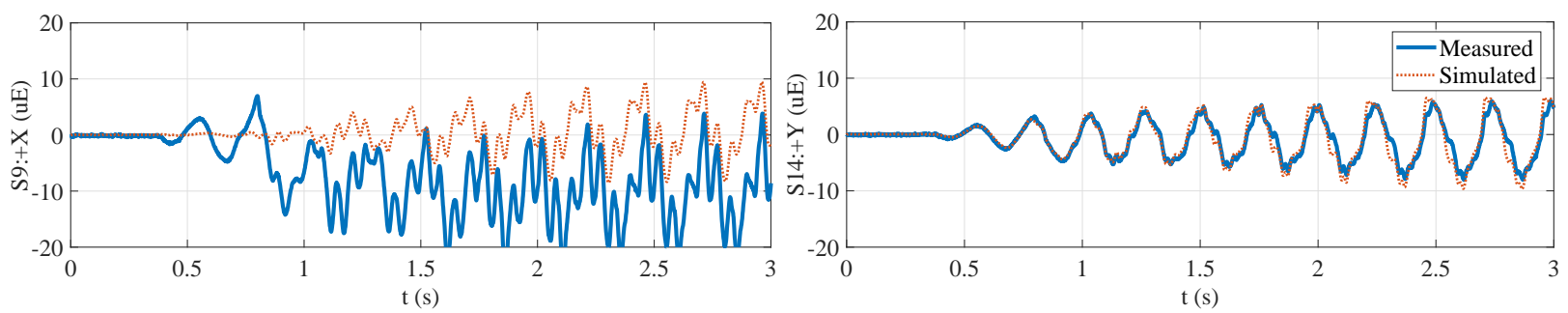

Figure 6: A visual inspection has been carried out to remove sensors according to step 3 of Table 3. S9:+X is an example of a discarded sensor, while $\mathrm{S} 14:+\mathrm{Y}$ is an example of a retained sensor.

The final selection step in point 4 of Table 3 allows to maximize the amount of information related to the estimated quantities that can be extracted from a given number of sensors. Following the approach used in [41] for non-linear 


\begin{tabular}{crll}
\hline Set Name & $M$ & Definition & Sensors list \\
\hline SS1 & 6 & Followed steps 1, 2, 3 and 4 of Table 3 & $\begin{array}{l}\text { S2:+Z, S10:+X, S11:+Y, S14:+Y, } \\
\text { S21:+Y, S22:+X }\end{array}$ \\
SS2 & 6 & $\begin{array}{l}\text { Followed steps 1, 2, 3 and 4 of Table 3. } \\
\text { Manually replaced sensor S21:+Y with } \\
\text { S31:+Y }\end{array}$ & $\begin{array}{l}\text { S2:+Z, S10:+X, S11:+Y, S14:+Y, } \\
\text { S22:+X, S31:+Y }\end{array}$ \\
& & \\
\hline
\end{tabular}

Table 4: Two different sensor sets have been defined to be employed as input to the estimation procedure.

systems, the selection is based on the definition of the following observability metric

$$
O_{L P}=\left(\operatorname{cond}\left(\left[\begin{array}{c}
\mathbf{A}_{L P}^{a}-\mathbf{I} \\
\mathbf{H}_{L P}^{a}
\end{array}\right]\right)\right)^{-1}
$$

where $\mathbf{A}_{L P}^{a}$ and $\mathbf{H}_{L P}^{a}$ are the system linearization matrices introduced in section 2 evaluated at the particular linearization point $L P . \mathbf{H}_{L P}^{a}$ is defined according to the sensors included in the sensor set as described in Appendix A. The matrix of which the condition number is evaluated in Eq. 42 is the one used in the Popov-Belovich-Hautus (PBH) observability test [42] evaluated for the eigenvalue 1 , which corresponds to the continuous-time eigenfrequency of $0 \mathrm{~Hz}$. Eigenvalue 1 is related to the random walk model of Eq. 29 and as explained in [9] it corresponds to the most difficult mode to observe. For this reason the focus is put on this eigenvalue and, as a consequence, only the observability of the unknown inputs vector $\mathbf{u}^{U K}$ is optimized.

As it appears clear from Eq. 42, the observability value $O_{L P}$ is specific for a given linearization point $L P$. In order to take into account a wider spectrum of operative conditions, several linearization points are considered. Those are generated by simulating the system model with the WCLs signals measured in experimental runs 1, 2 and 3 of Table 2 and taking the linearizations at time equal to $0 \mathrm{~s}, 1 \mathrm{~s}, 2 \mathrm{~s}, 3 \mathrm{~s}, 4 \mathrm{~s}$ and $5 \mathrm{~s}$. Six values of observability are in this way obtained for each of the three training scenarios. The overall observability value is then taken as

$$
O=\sum_{T S=1}^{3} \bar{O}^{T S}
$$

where $\bar{O}^{T S}$ is defined as $\sum_{k=1}^{6}\left(O_{T S, k} / 6\right)$, hence the latter being the average of the observability values over the same training scenario $T S$.

The $M$ sensors which maximize the overall observability value $O$ defined in Eq. 43 are hence selected among the remaining sensors after steps 1,2 and 3 of Table 3.

Following this procedure, two sensors sets have been obtained, i.e. $\mathbb{S S 1}$ and $\mathbb{S}$ 2, cf. Table 4 and Figure 7. Both sensor sets contain the theoretical minimal number of 6 sensors to ensure observability. Indeed, the theoretical minimal number of position level sensors that have to be used in order to ensure observability of the unknown inputs is equal to the number of unknown inputs as outlined in [9]. As in this work six inputs, i.e. the WCLs, have to be estimated along with the system states, all sensor sets have to include at least 6 strain gauges. Sensor set $\mathbb{S S} 1$ has then been defined by following the steps of Table 3, while sensor set $\mathbb{S S} 2$ has been generated starting from $\mathbb{S S} 1$ and by manually replacing sensor S21:+Y with S31:+Y. Although S31:+Y is quite closely located to S21:+Y, the latter is indicated by the selection step 4 of Table 3 while the former is discarded. $\mathbb{S} 22$ has been introduced to show the usefulness of taking into account observability for the sensor selection as clarified with the results of Subsection 5.3.

\subsection{On the load cell use}

One of the objectives of this paper is to propose a procedure which can estimate input loads on a mechanical system, without directly measuring them. However a load cell is used for the sensor selection procedure as explained in the previous Subsection 4.1. This is done with the purpose of generating a reference case in which the best possible performances of the method can be assessed in terms of accuracy of the estimation. 

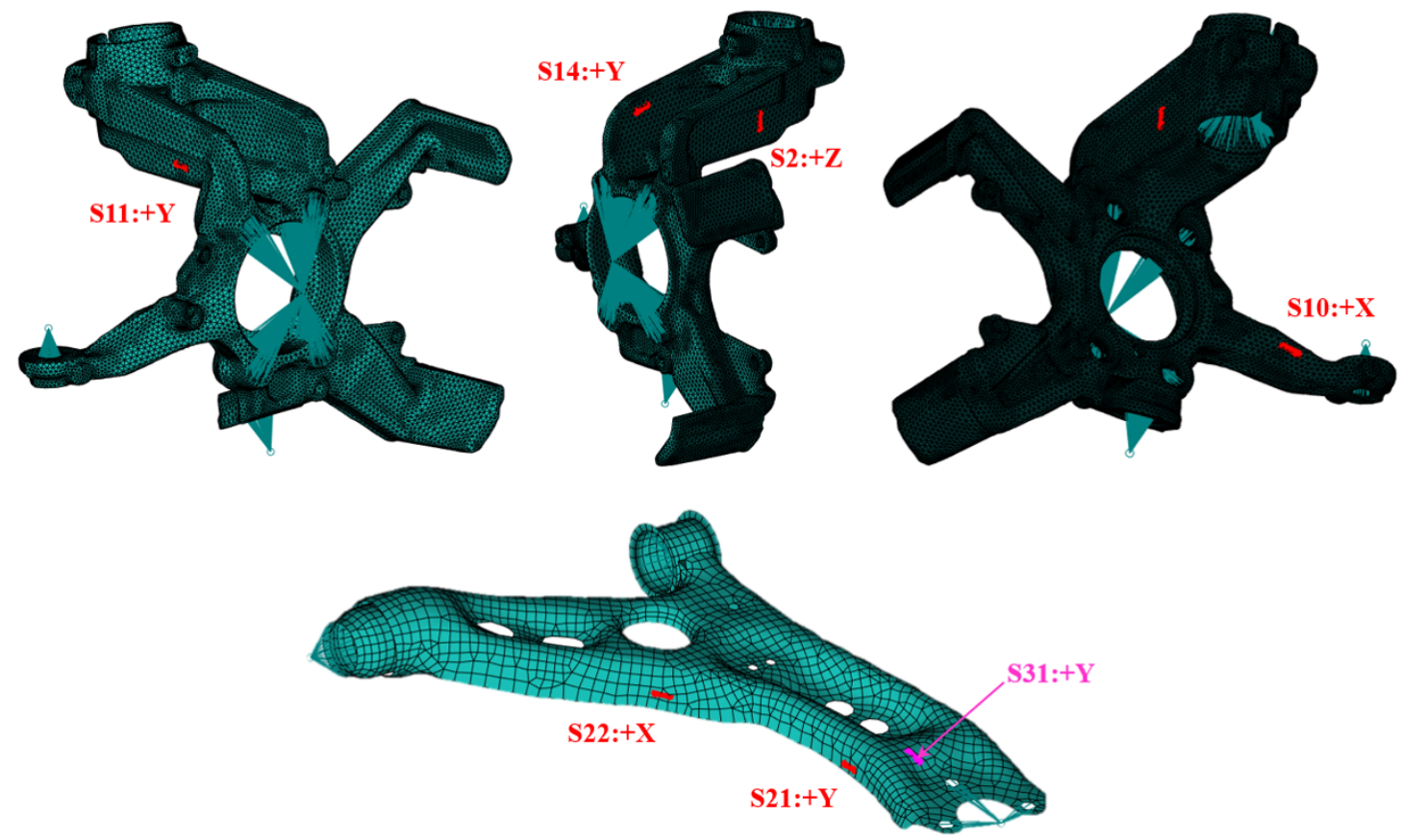

Figure 7: Sensor sets listed in table 4. The six sensors of sensor set $\mathbb{S S 1}$ are shown in red, while sensor S31:+Y which replaces $\mathrm{S} 21:+\mathrm{Y}$ in sensor set $\mathbb{S S} 2$ is shown in magenta.

As it is clear from the validation results reported in Section 5, the accuracy of the method in the reconstruction of input loads is strictly related to the accuracy of the model in simulating the response of the sensors of the employed sensor set. The use of the load cell then plays an important role in assessing such model accuracy in step 3 of Table 3. This step can also be seen as a calibration step that is inserted in order to guarantee a certain accuracy level. If a load cell is not available for this assessment, some other procedure could be put in place in which a known input load is applied and the responses are measured even in purely static conditions, e.g. by making use of dead weights.

Concerning instead selection steps 2 and 4 of Table 3, the use of a load cell is not strictly necessary. For step 2 indeed synthetic load signals resembling those of experimental run 2 of Table 2 could be generated and applied to the simulation model, which would probably lead to similar results of the ones exposed in the previous Subsection 4.1 in terms of rejected sensors. Similarly for step 4, synthetic input loads representative of operational conditions could be generated and used, which would probably again lead to similar results in terms of optimal sensor selection.

\section{Validation results}

The estimation procedure proposed in Section 2 is applied in this section to the McPherson suspension system of Section 3 in order to concurrently estimate the six WCLs and the full strain field over the steering knuckle and the control arm. Results related to the experimental runs of Table 2 obtained with the sensors sets of Table 4 are first displayed and discussed in Subsections 5.1, 5.2 and 5.3, while more details about the setting of parameter $Q_{u}$ are provided in Subsection 5.4.

Anyway, before presenting the results, some important details about the setting of $\mathbf{Q}_{k}, \mathbf{Q}_{k}^{U K}$ and $\mathbf{R}_{k}$ are given. As already mentioned in Section 2, the noise processes $\mathbf{w}_{k}, \mathbf{w}_{k}^{U K}$ and $\mathbf{v}_{k}$ are assumed to be white, zero-mean and uncorrelated, but in the majority of cases such assumptions are not realistic especially for what concerns with $\mathbf{w}_{k}^{U K}$ as the unknown inputs $\mathbf{u}_{k}^{U K}$ has in many cases different characteristics. Anyhow, the unknown inputs in vector $\mathbf{u}_{k}^{U K}$ are assumed here to be the main source of uncertainty in the model equations, i.e. the first expression in Eq. 27 and Eq. 29. For this reason all the elements of matrix $\mathbf{Q}_{k}$ are set to zero, while $\mathbf{Q}_{k}^{U K}$ is set to be a diagonal matrix with diagonal entries all equal to the non-zero value $Q_{u}$. The implications of this assumption can be understood from the 
results of the following subsections. Matrix $\mathbf{R}_{k}$ is then set as a diagonal matrix with diagonal entries estimated from the relative measurement channels, as $\mathbf{v}_{k}$ corresponds to the noise in the measurement chain. $Q_{u}$ therefore remains the only arbitrary parameter of the estimation procedure. In Subsection 5.4 it is shown that for the system under examination, a lower bound for $Q_{u}$ above which all estimations converge to the same result can be found. Such value equal to 10 is selected for the estimations presented in the other subsections. The measurement unit for $Q_{u}$ is omitted for conciseness as it is omitted in the following subsections, but it would correspond to $N^{2}$ when the related quantity is a force and to $(\mathrm{Nm})^{2}$ when the related quantity is a moment.

The way of setting the parameters $\mathbf{Q}_{k}, \mathbf{Q}_{k}^{U K}$ and $\mathbf{R}_{k}$ just explained, can hence be use to generate an automatic parameters selection procedure without the need of inputs provided by the user of the estimation procedure and for this reason has been adopted.

\subsection{Both inputs and outputs are accurately estimated when the system model incorporates accurate input-output relations}

Figure 8 shows the inputs estimation results related to the WCLs for the first 4 seconds of experimental run 2 of Table 2 which corresponds to a sinusoidal motion of the hydraulic shaker at $4 \mathrm{~Hz}$ with $5 \mathrm{~mm}$ of amplitude in the vertical direction. The estimated signals have been obtained by making use of sensor set $\mathbb{S} 1$ in the estimation procedure and are compared with the ones directly measured with the load cell.
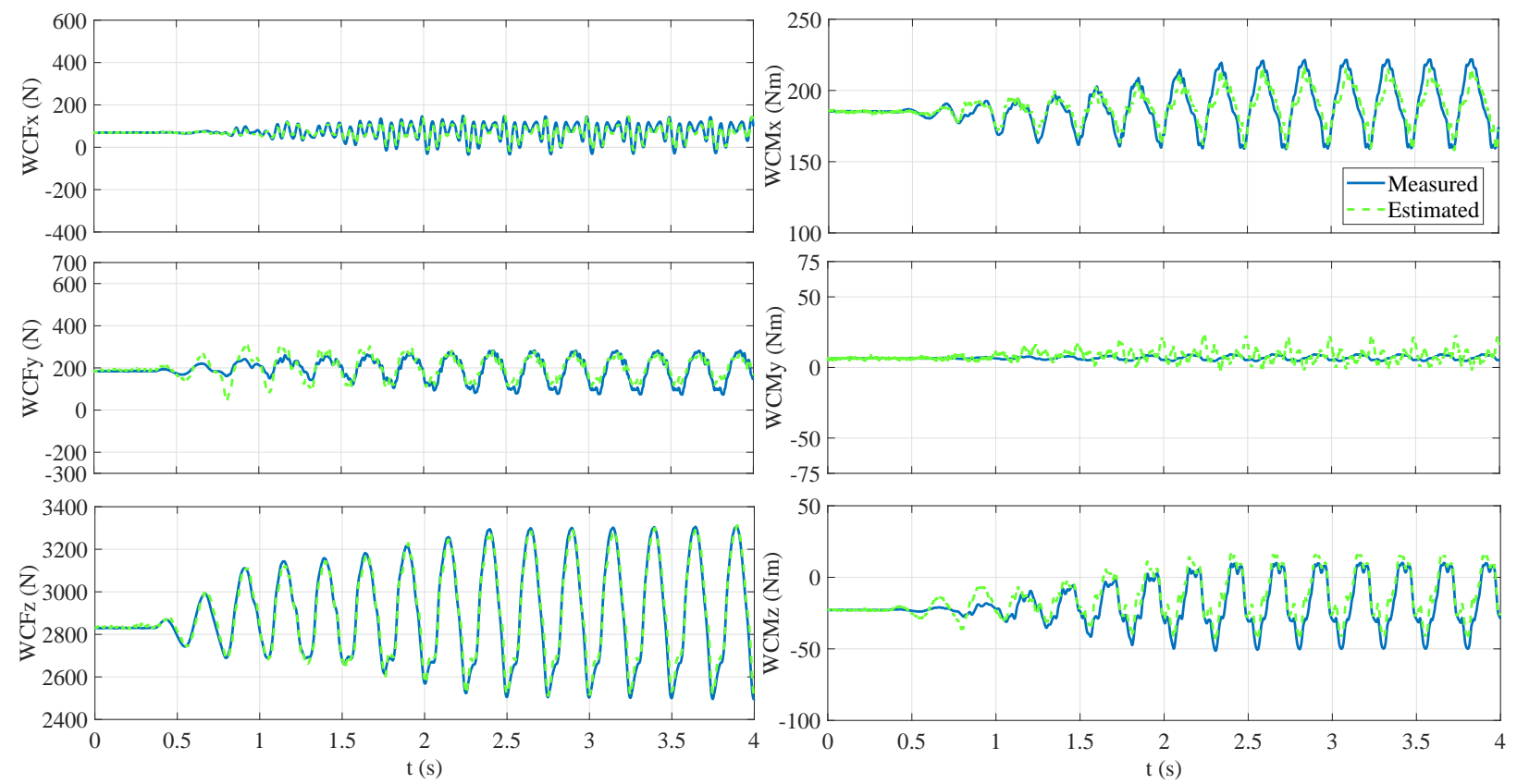

Figure 8: All six Wheel Center Loads, i.e. three Wheel Center Forces (WCFs) and three Wheel Center Moments (WCMs), can be accurately estimated for experimental run 2 of Table 2.

An excellent matching between estimated and measured signals can be observed for all six inputs, especially when looking at a zoom on the same results reported in Figure 9. Only WCMy shows a relatively weak match, but this load has a very low amplitude. Indeed it corresponds to the moment around the rolling axis of the tire and as explained in Section 3 a rolling movement between the tire and the steering knuckle is allowed by the load cell. Information related to such load is then also limited in the sensors, meaning that the response observable in the latter and due to such load is smaller than the noise. Therefore the reconstruction quality for such load is worse. Anyway such load is correctly estimated to be the one with the smallest amplitude among the moments.

As explained in Section 4 sensors of $\mathbb{S} 1$ are accurate in the sense that the matching between the measured values and the corresponding ones obtained by forward simulation applying the measured WCLs is like the one of the example reported on the right side of Figure 6. Estimation results representative for all sensors of $\mathbb{S} \mathbb{S} 1$ are reported in 

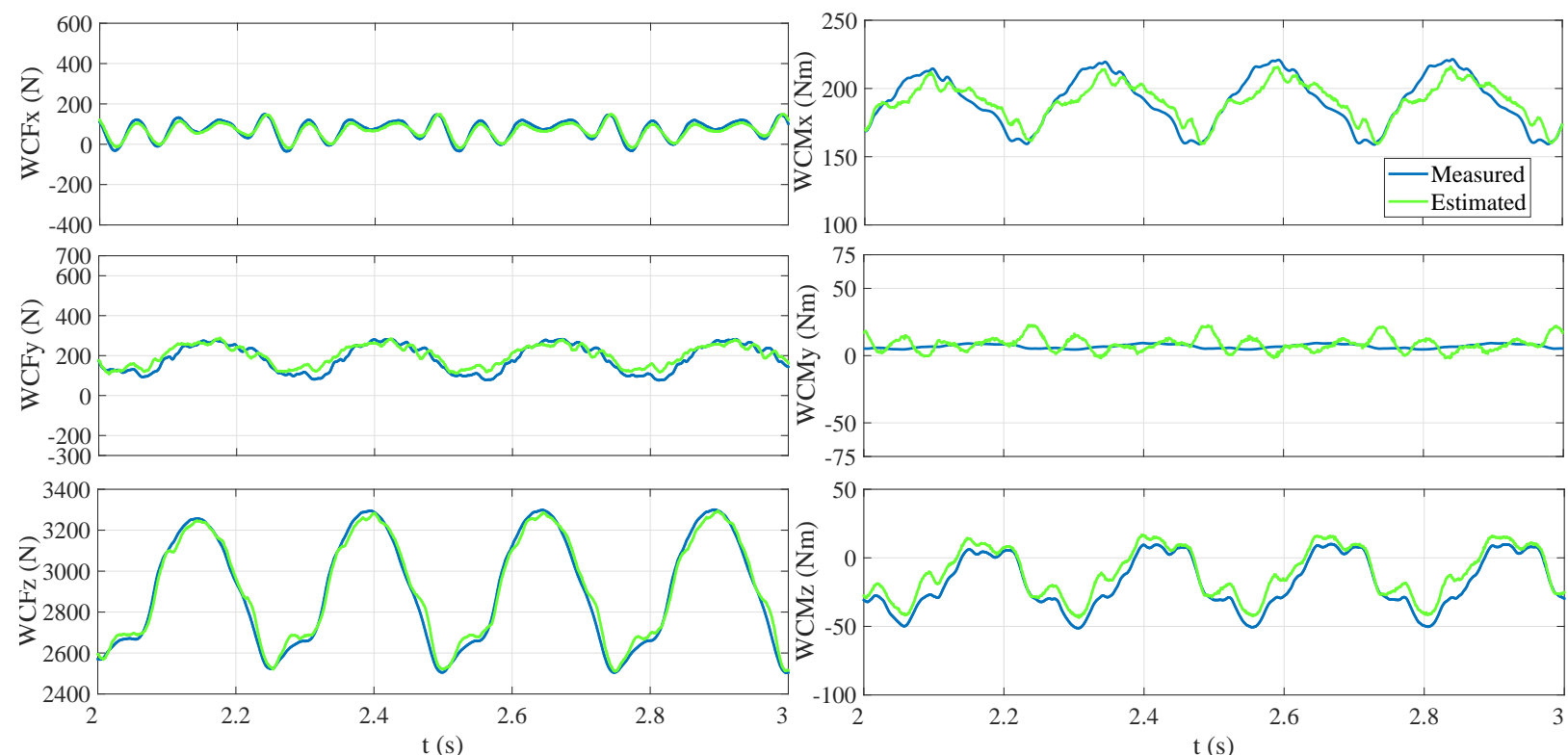

Figure 9: The zoom on the results shows the excellent quality of the signals reconstruction in comparison with the load cell validation signals for the six Wheel Center Loads, i.e. three Wheel Center Forces (WCFs) and three Wheel Center Moments (WCMs). Only WCMy shows a relatively weak match matching, but this load has a very low amplitude as the tire is allowed to roll in the set up.

Figure 10 and a zoom on the same results is reported in Figure 11. It can be observed how the estimation procedure corrects the strain values to be very close to the measured ones. The estimated signals result then to be much closer to the measured signals than the ones obtained by forward simulation with known WCLs also reported on the figure. This is a consequence of the way in which the parameters $\mathbf{Q}_{k}, \mathbf{Q}_{k}^{U K}$ and $\mathbf{R}_{k}$ have been set as explained in the beginning of this section. More in detail the value of $Q_{u}$ which fills in the diagonal entries of $\mathbf{Q}_{k}^{U K}$, i.e. 10 , is much higher than the diagonal entries of $\mathbf{R}_{k}$ which are based on the sensors noise hence with order of magnitude of $10^{-14} \mu \epsilon^{2}$.
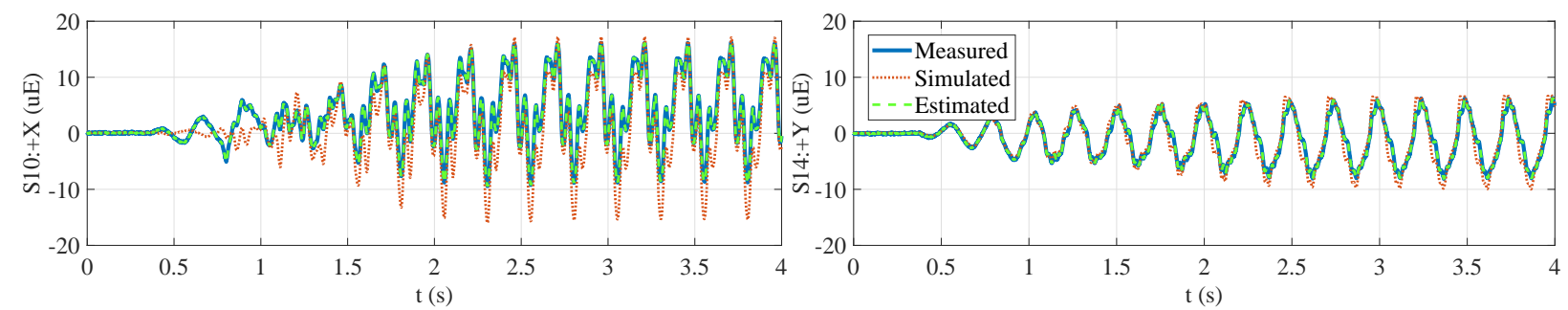

Figure 10: All six sensors employed in the estimation ( $\mathbb{S S} 1$ of table 4) exhibit a very good matching between simulation and experiments (only S10:+Y and S14:+Y are displayed for conciseness). This is one of the necessary conditions to obtain an accurate input reconstruction.

An overview of the strain estimation results along with a comparison with simulation results obtained by directly applying the measured WCLs to the system model is instead reported in Figure 12. Both estimated and simulated results are reported in terms of the Averaged Absolute Error (AAE) defined as $\sum_{i=1}^{T}\left|s_{\text {valid,i } i}-s_{\text {measur, } i}\right| / T$, where $s_{\text {measur,i }}$ is the $i$-th measured signal sample, $s_{\text {measur, } i}$ is the $i$-th validation signal sample - which can be estimated or simulated, and $T$ is the total number of samples. The sensors are ordered on the horizontal axis according to the measured MRA also reported on the figure. It can be observed how the AAE for the estimated signals remains rather constant over the different locations and with respect to the MRA. The same holds for the AAE related to the simulation signals. The overall quality of the strains estimation is therefore comparable to the quality of the strains simulation. The main 

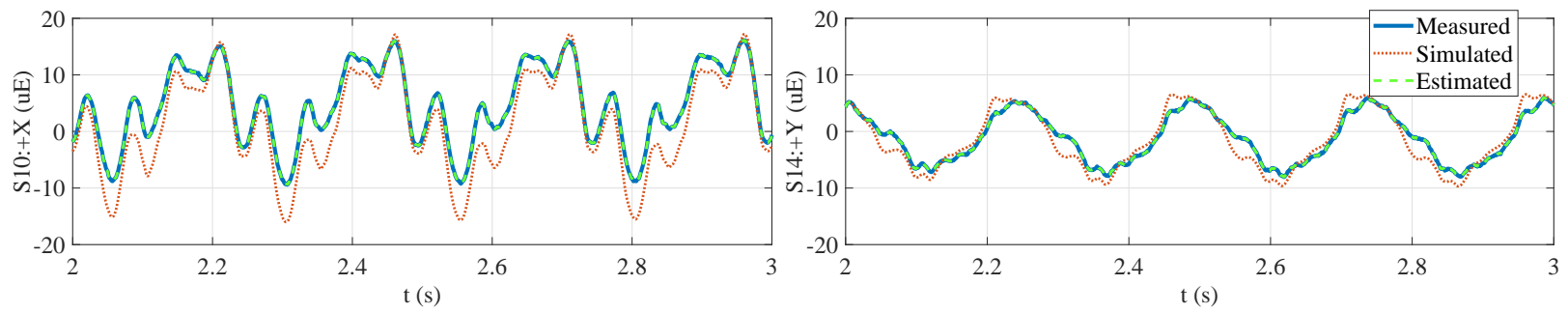

Figure 11: The zoom on the results for the sensors employed in the estimation shows how the estimation procedure corrects the related strain signals to be very close to the measured signals.

differences are related to the sensors of $\mathbb{S S} 1$ as the estimation procedure improves the matching with the measured signals. Figure 13 shows a few examples of strain signals not included in $\mathbb{S S} 1 . \mathrm{S} 16:+\mathrm{Y}$ and S15:+X are representative of most of the sensors in terms of quality of the reconstruction. For S16:+Y not much difference is observable between simulated and estimated signals which are both very close to the measured ones. For S15:+X a successful corrective action of the estimation procedure implies the estimated signal to be closer to the measured one than the simulated signal. This can be explained as $\mathrm{S} 15:+\mathrm{X}$ is closely located to $\mathrm{S} 10:+\mathrm{X}$ which is part of $\mathrm{SS} 1$. However such a corrective action is not always exhibited. For S9:+X for instance both estimated and simulated signals are quite distant from the measured one. S9:+X is very closely located to a connection point between steering knuckle and tierod, hence local effects which cannot be properly modeled might have a strong impact. A few other points show a reconstruction quality comparable to the one of S9:+X. In other few cases the signal can be properly tracked over time, but the amplitude estimation is slightly inaccurate as in the case of S31:+Y.
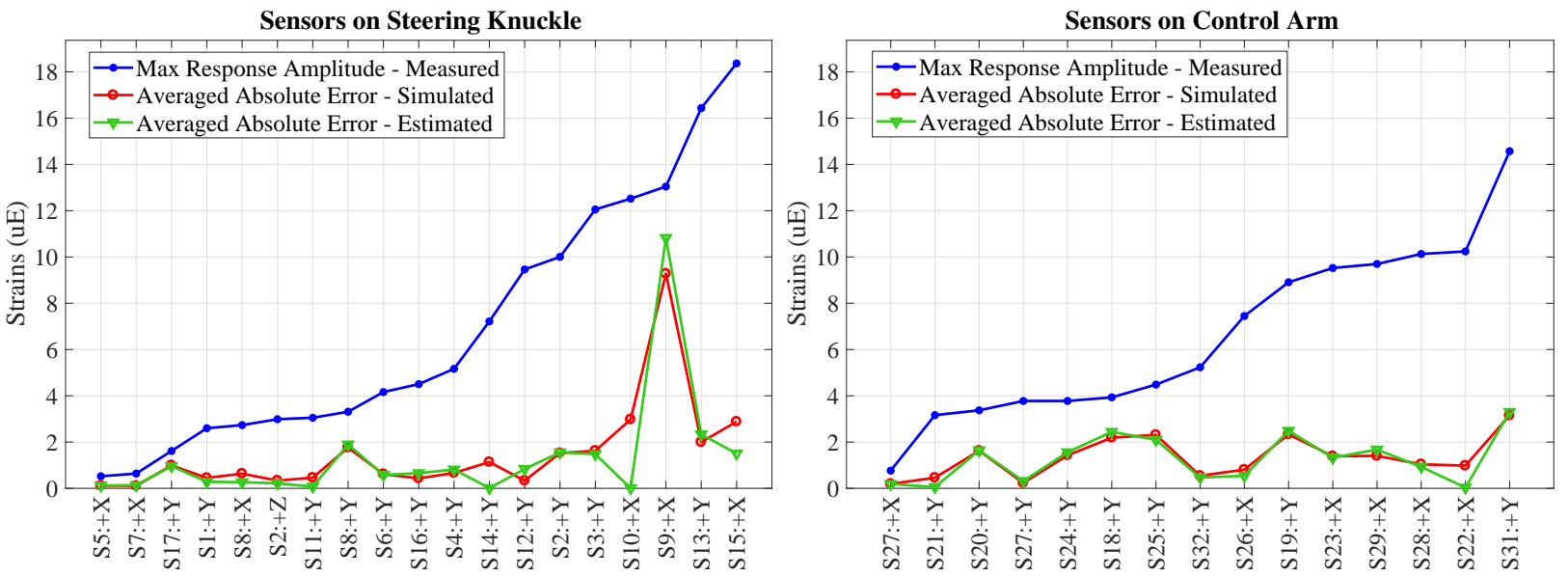

Figure 12: The overall quality of the strain estimation is comparable to the quality of the strain simulation and the estimation error remains nearly constant over the different locations.

It can hence be concluded that an excellent input estimation quality can be achieved with the estimation procedure proposed in Section 2 if the system model employed incorporates accurate Input-Output (I-O) relations between input to be estimated and output used in the estimation procedure. In particular, a forward simulation of the system model with known input loads should achieve a matching of the outputs used in the estimation procedure like the one exhibited in the examples of Figure 10. The full strain field can then also be estimated with an accuracy which is at least as good as the one achievable with forward simulation by applying the measured WCLs to the model. As further demonstrated in Subsection 5.3, the requirement on the simulation model accuracy represents only a necessary but not a sufficient condition as the sensor selection step 4 of Table 3 based on observability also plays a crucial role. 

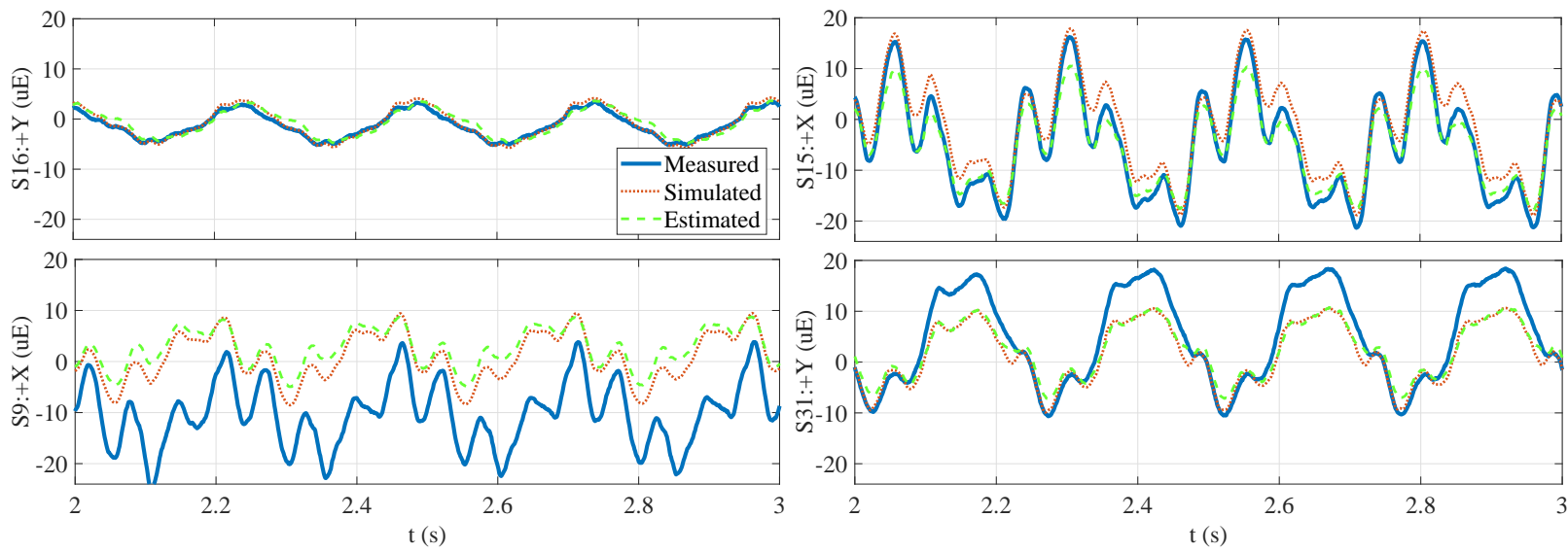

Figure 13: Examples of strain locations not employed in the estimator. The strain estimation examples for S16:+Y and S15:+X, representative of most of the other locations, show the good signal reconstruction quality obtained. S9:+X is instead an example of sensors very closely located to a connection point where local effects which cannot be properly modeled might have a strong impact. S31:+Y is an example of sensor for which the signal can be properly tracked over time, but the amplitude estimation is slightly inaccurate.

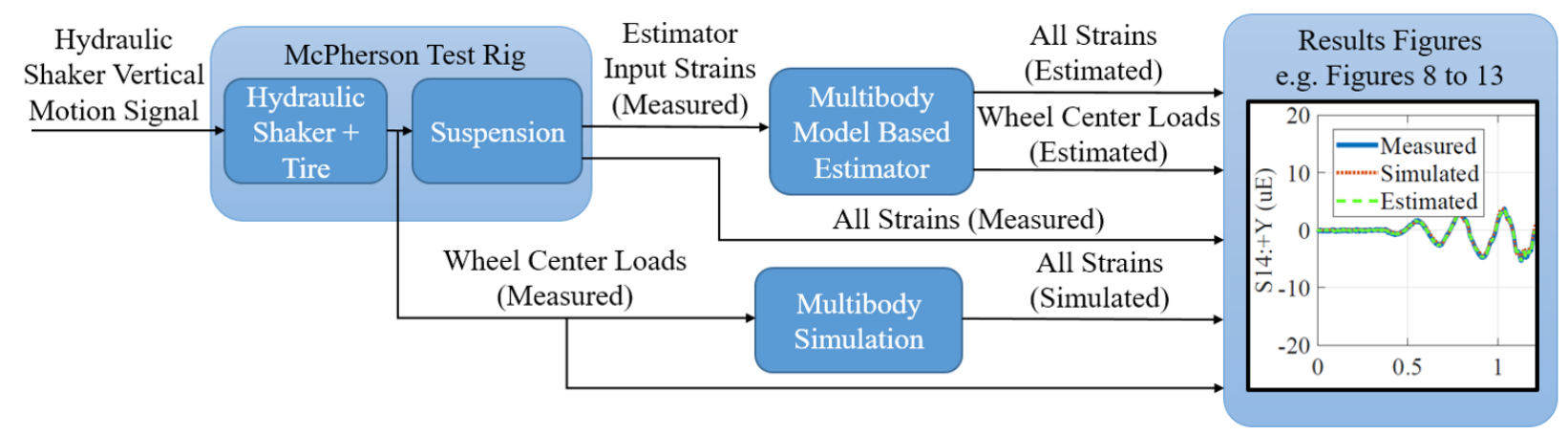

Figure 14: The diagram visualizes and summarizes how the time signals shown in the results figures have been retrieved.

\subsection{Modeling inaccuracies affect only the inputs estimation while the outputs estimation remains accurate}

Figure 15 shows the inputs estimation results related to the WCLs for the first 4 seconds of experimental run 4 of Table 2 which corresponds to the road profile reproduction. The estimated signals have been obtained by again making use of sensor set $\mathbb{S S 1}$ in the estimation procedure and are compared with the ones directly measured with the load cell.

All the six loads can be concurrently estimated, even though the estimation accuracy has degraded with respect to the one observed in the results of the previous Subsection 5.1 as it appears more evident when looking at the zoom on the same results reported in Figure 16. This is mainly true for $W C M x$ for which the amplitude is slightly overestimated and for $W C F y$ for which a phase shift can be noted. $W C F x, W C M z$ and $W C F z$ are still very accurately estimated. $W C M y$ is correctly estimated to be the one with the smallest amplitude among the moments.

Such degradation of the loads estimation accuracy can be explained by looking at Figure 17 which shows signals related to the six sensors of sensor set $\mathbb{S S} 1$. It can be seen that despite the matching between measured and simulated signals remains relatively strong for 4 of the 6 sensors, the same does not hold for S11:+Y and S21:+Y. More in detail the simulated amplitude values for $\mathrm{S} 11:+\mathrm{Y}$ are considerably higher than the measured ones, while a clear phase shift between simulated and measured signals can be observed for S21:+Y. Such discrepancies, that can be related to specific un-modeled phenomena that occur for this specific experimental run, are then corrected by the estimation procedure as visible on the figure. However, as a result, the inputs estimation quality degrades. This is partially due 

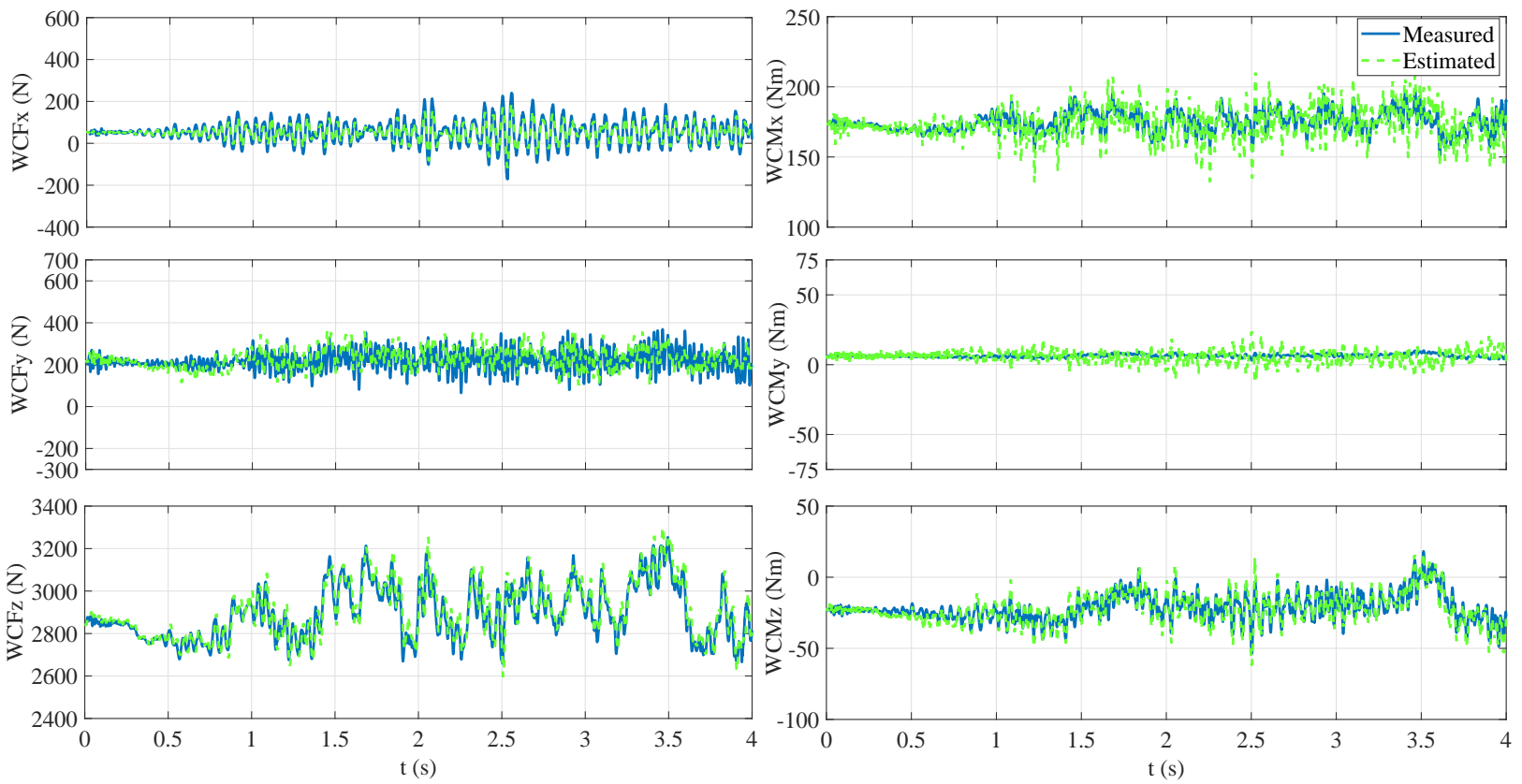

Figure 15: The six Wheel Center Loads, i.e. three Wheel Center Forces (WCFs) and three Wheel Center Moments (WCMs), can be estimated also for experimental run 4 of Table 2, but the estimation quality degrades for some components.
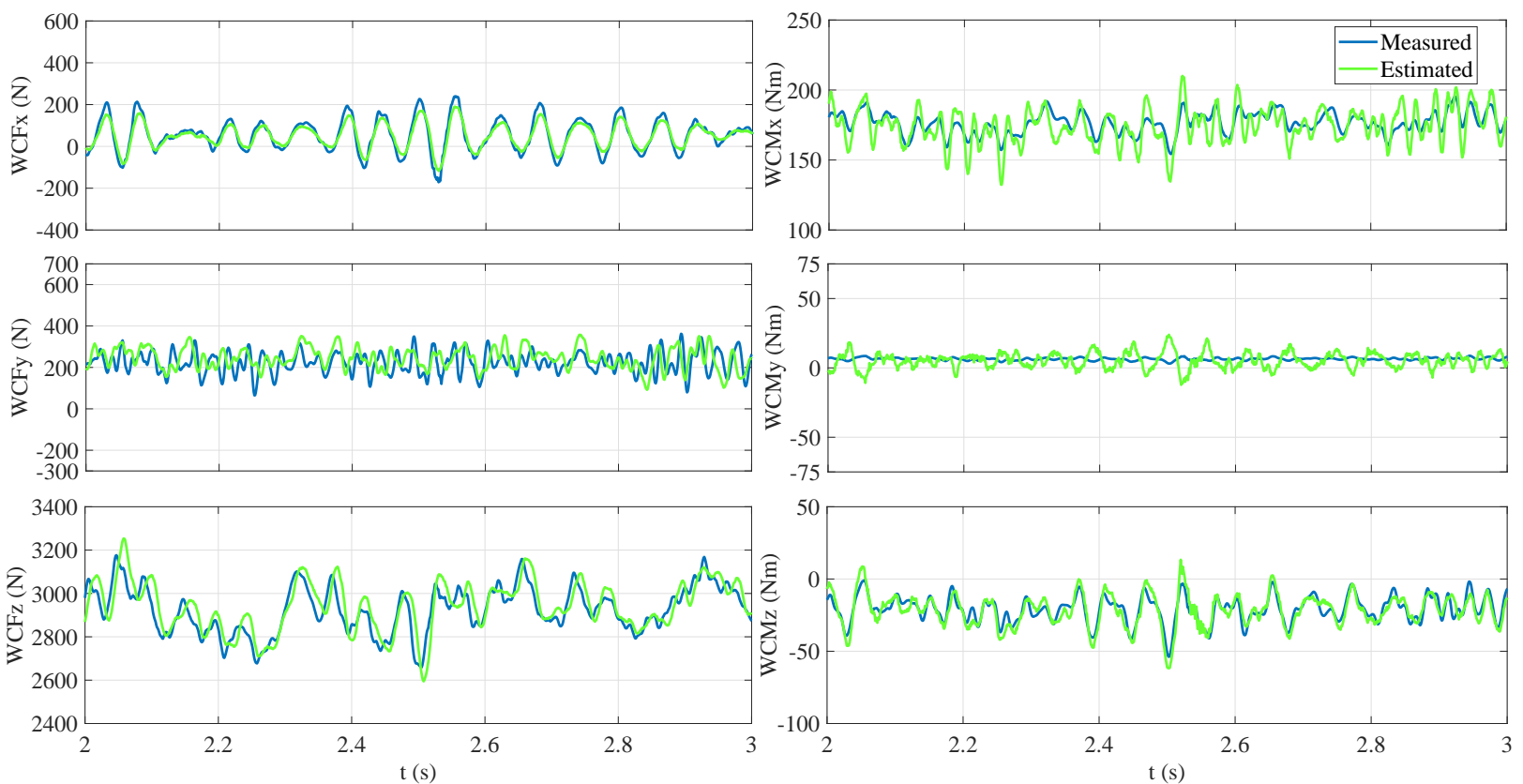

Figure 16: Zoom on the results for the Wheel Center Loads, i.e. three Wheel Center Forces (WCFs) and three Wheel Center Moments (WCMs). Even though $W C F x, W C F z$ and $W C M z$ are still accurately estimated, a considerable phase shift appears for $W C F y$ while the amplitude of WCMx is slightly overestimated.

to the way in which the parameters $\mathbf{Q}_{k}$ and $\mathbf{Q}_{k}^{U K}$ have been set as explained in the beginning of this section. More in detail to the setting of $\mathbf{Q}_{k}$ to zero which, in combination with the setting of $\mathbf{Q}_{k}^{U K}$ to non-zero values, leads to a higher 
tendency of the estimation procedure to correct the values of $\mathbf{u}_{k}^{U K}$ rather than the values of $\mathbf{x}_{k}$.
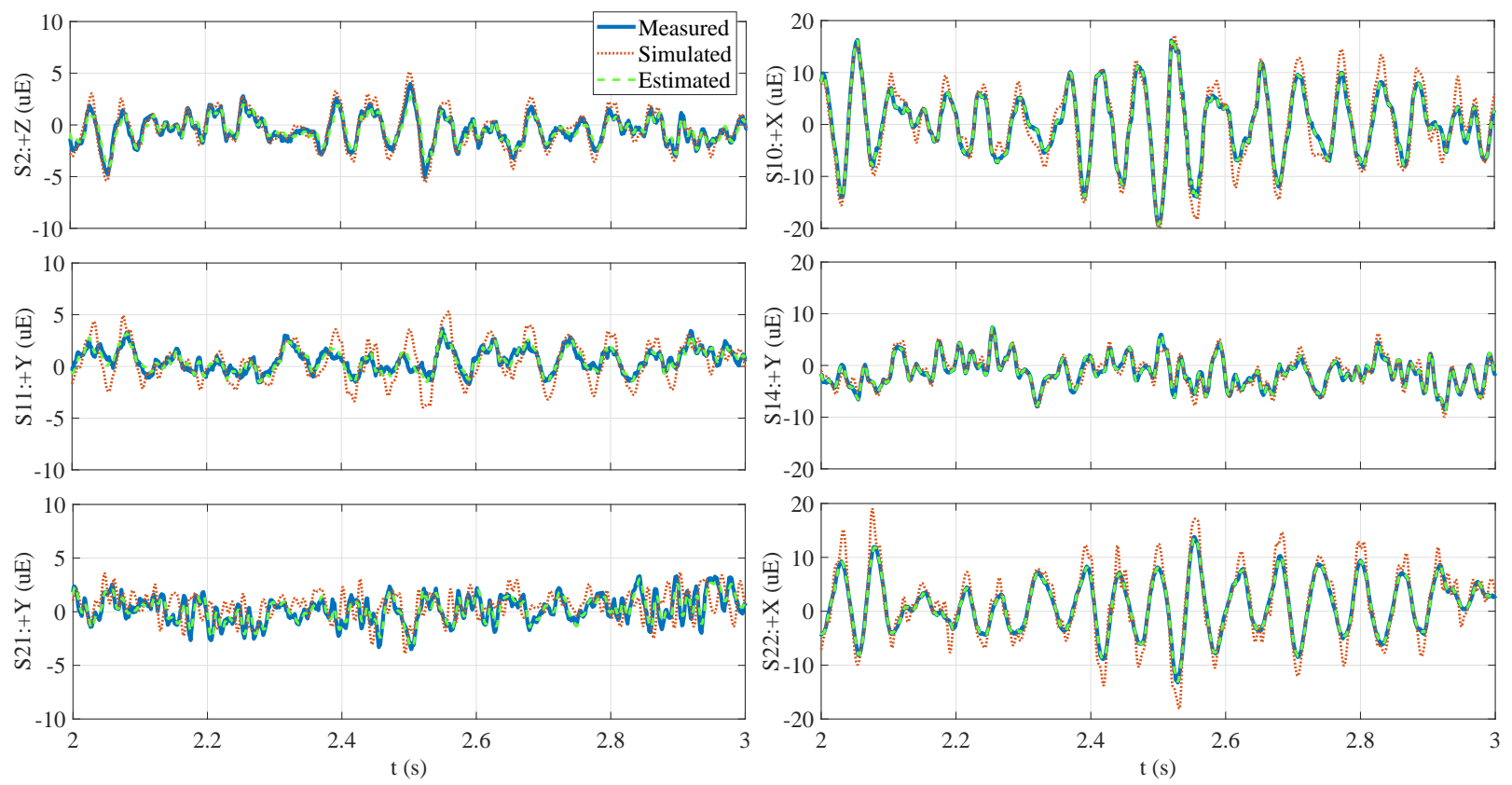

Figure 17: Results for the sensors employed in the estimation ( $\mathbb{S S} 1$ of table 4). The mismatching between simulated and measured strain signals for S21:+Y and S11:+Y explains the input estimation quality degradation. However all the six strain signals are corrected by the estimator in order to better match the measured values.
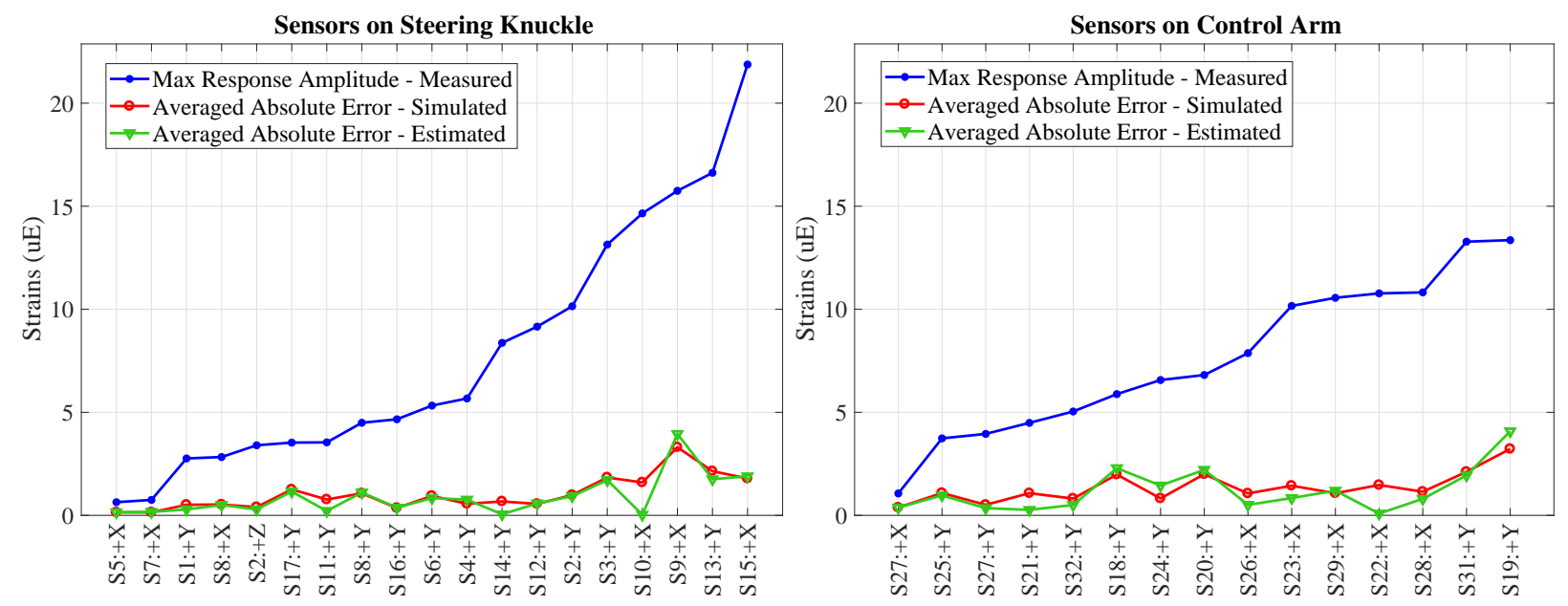

Figure 18: As a result of the estimator action which corrects the states in order to match the measured strains, the estimation of the whole strain field is as accurate as in the results of the previous Subsection 5.1.

On the other hand, thanks to this correction, the overall strains estimation quality is again similar to quality of the strains simulation where the WCLs are measured and applied as known inputs to the model, as in the case of the results reported in the previous Subsection 5.1 where also the input reconstruction is very accurate. This can be observed by looking at the overview of the strain estimation results in terms of AAE reported in Figure 18, along with a comparison with the corresponding simulation results. The sensors are ordered on the horizontal axis according to 

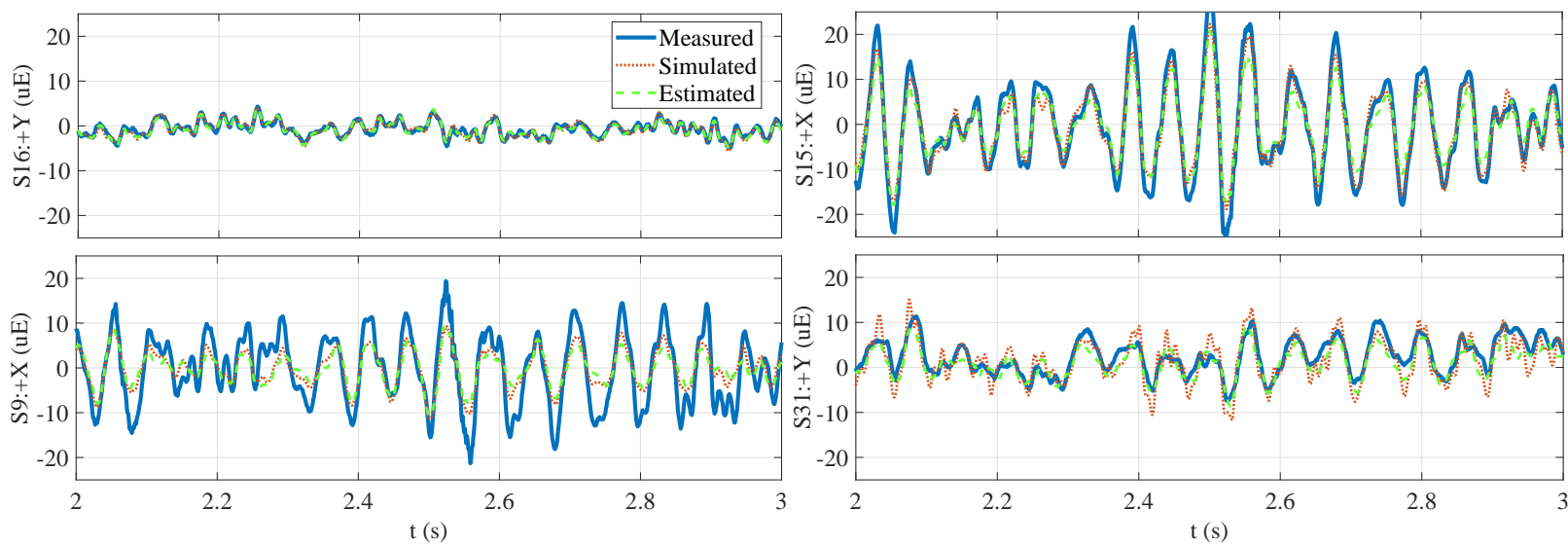

Figure 19: Examples of strain locations not employed in the estimator. As in the results of the previous Subsection 5.1 most of the strains are accurately estimated as in the examples represented by S16:+Y and S15:+Y. S9:+X and S31:+Y are again examples of sensors which report among the highest values of averaged absolute error.

the measured MRA also reported on the figure. Note that the resulting sensors order is slightly different from the one of Figure 12, as it has been retrieved according to the MRA values related to a different experimental run. Figure 19 shows a few examples of strain signals not included in $\mathbb{S S 1}$. S16:+Y and S15:+X are representative of most of the sensors in terms of quality of the reconstruction. S9:+X and $\mathrm{S} 31:+\mathrm{Y}$ are again examples of sensors which report among the highest values of AAE.

It can hence be concluded that modeling inaccuracies, which result in less accurate I-O relations between inputs to be estimated and outputs used in the estimation, mainly deteriorate the inputs estimation quality while do not affect the output estimation quality.

The results are also reported in Figure 20 and Figure 21 in the frequency domain in terms of Power Spectral Densities (PSDs), for frequencies up to $100 \mathrm{~Hz}$ as above this value the Signal-To-Noise ratios of the sensors in not sufficient. More in detail Figure 20 shows the PSDs of the WCLs from which it is visible that a relatively good reconstruction can be achieved for $W C F x, W C F z, W C M x$ and $W C M z$ until approximately $40 \mathrm{~Hz}$. Figure 21 instead shows two examples of sensors of $\mathbb{S S} 1$, i.e. S10:+X and $\mathrm{S} 14:+\mathrm{Y}$, from which it is visible that the correction action of the estimation procedure is active in the full frequency spectrum shown. On the same figure two examples of sensors not included in SS1, i.e. S16:+Y and S31:+Y, are also reported, from which it is visible that the amount of correction due to the estimation procedure depends on the sensor and on the frequency.

\subsection{The availability of a physically representative model allows to optimally place the sensors, hence avoiding poor observability of the quantities of interest}

Figure 22 shows inputs estimation results related to WCFy and WCMx in terms of PSDs for experimental run 4 of Table 2 which corresponds to the road profile reproduction. In this case a comparison between results obtained by making use of sensor set $\mathbb{S S} 1$ and results obtained by making use of $\mathbb{S S} 2$ is reported. As explained in Section 4 sensor set $\mathbb{S S} 2$ differs from sensor set $\mathbb{S S} 1$ because of the replacement of S21:+Y with S31:+Y. S21:+Y and S31:+Y are closely located to each other and are both approximately aligned with the lateral direction, although only the former is selected by the optimal sensor placement step 4 of Table 3 while the latter is discarded.

Results related to $W C F x, W C F z, W C M y$ and $W C M z$ are not reported for conciseness as only minor differences are observable between estimated signals obtained by making use of $\mathbb{S S} 1$ and the ones obtained by making use of $\mathbb{S S} 2$ for these loads. Large differences are instead visible by looking at the results reported for WCFy and WCMx in the low frequencies region, especially between $0 \mathrm{~Hz}$ and $20 \mathrm{~Hz}$. If looking at the location and orientation of S21:+Y and S31:+Y in Figure 7, it appears clear that these sensors should be the ones discriminating between WCFy and WCMx. As shown by the results of Figure 22, a non-optimal placement of such sensors may jeopardize the estimation accuracy. On the other hand the availability of a simulation model allows to run an optimal sensor placement analysis as the one of step 4 in Table 3, and to correctly estimate the quantity of interest as happened for sensor set $\mathbb{S} \mathbb{S 1}$ in Subsection 5.1. 

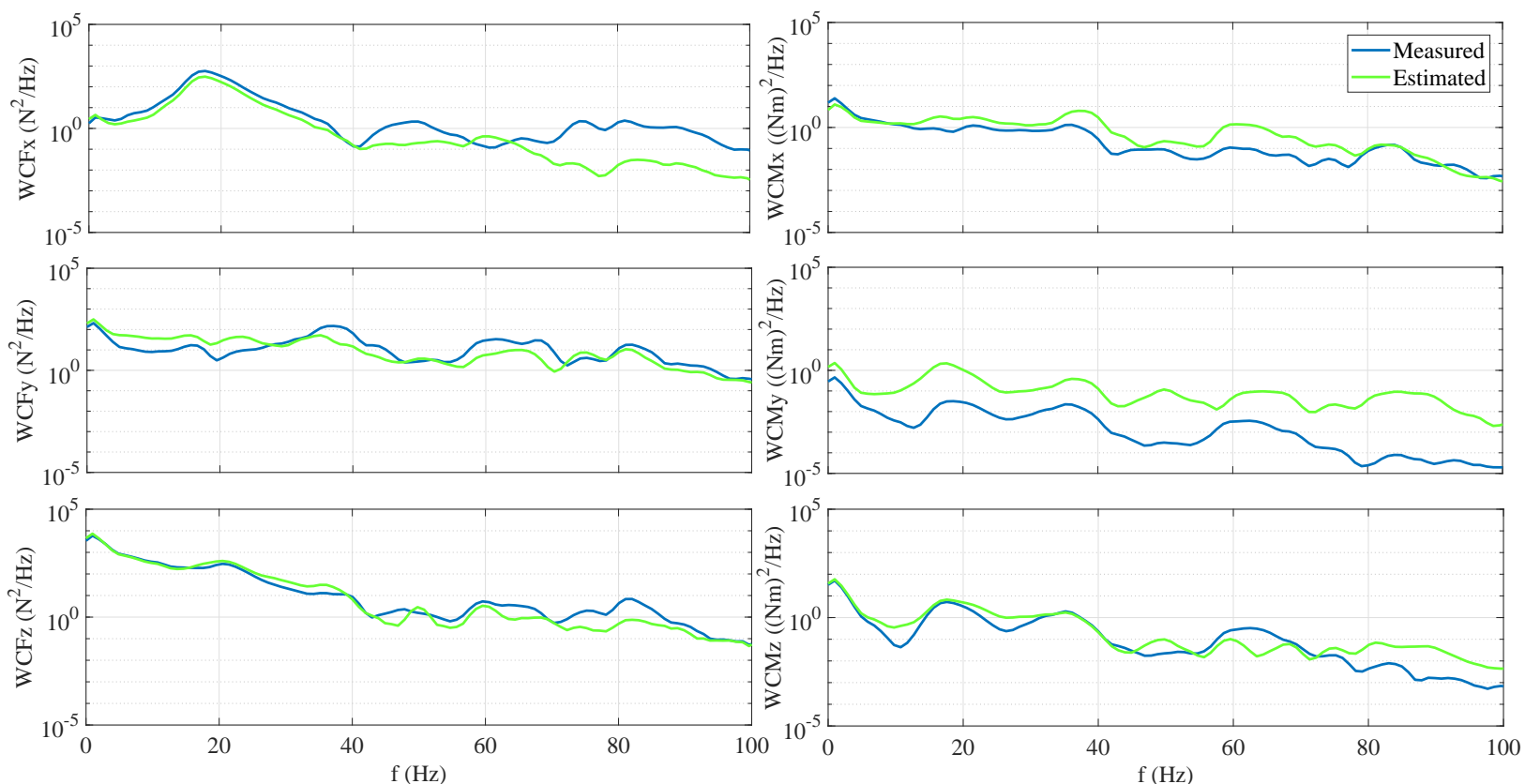

Figure 20: Power Spectral Densities (PSDs) results for the Wheel Center Loads, i.e. three Wheel Center Forces (WCFs) and three Wheel Center Moments (WCMs). From the PSDs of the signals it can be observed that relatively good reconstruction can be achieved for $W C F x, W C F z, W C M x$ and $W C M z$ until approximately $40 \mathrm{~Hz}$.
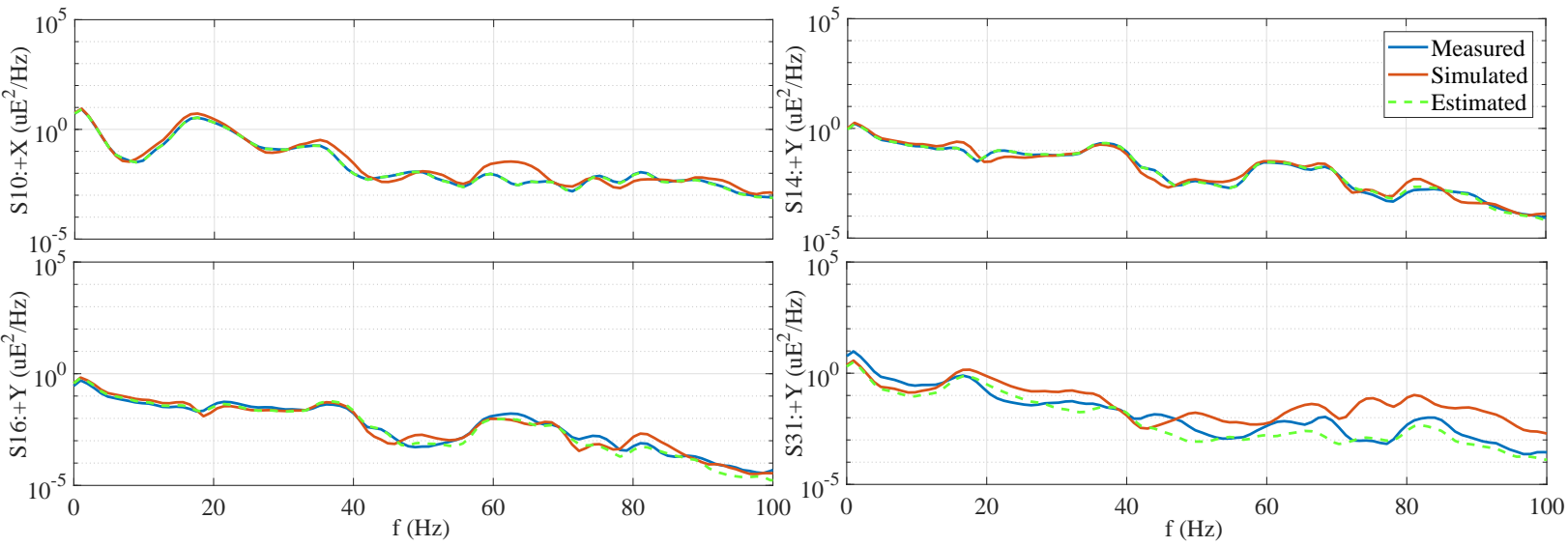

Figure 21: For the sensors of $\mathbb{S S} 1$ (employed in the estimator, see table 4), e.g. S10:+X and S14:+Y, it can be observed how the corrective action of the estimation procedure is active in the full frequency spectrum shown. For sensors not included in $\mathbb{S S} 1$ instead, e.g. S16:+Y and S31:+Y, the amount of correction due to the estimation procedure depends on the sensor and on the frequency. 

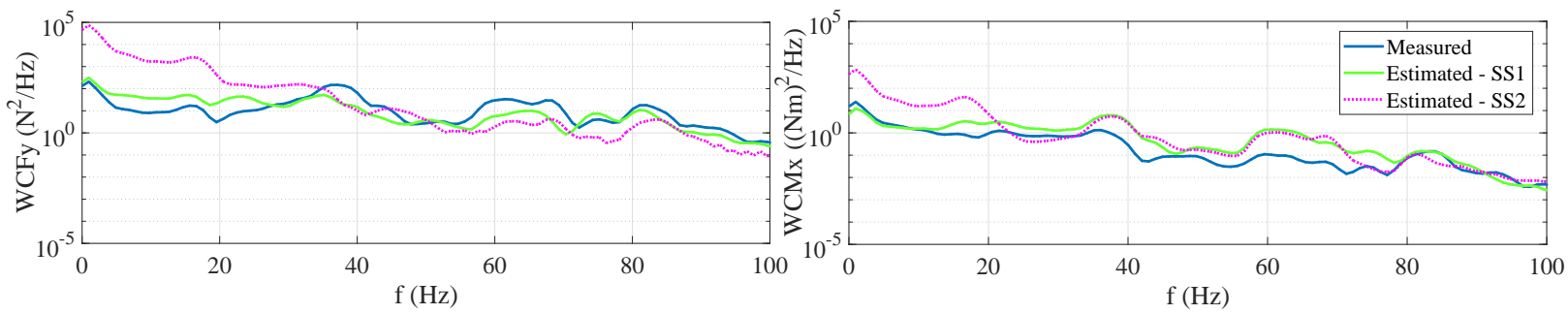

Figure 22: Loads WCFy and WCMx cannot anymore be correctly observed if sensor S21:+Y of the optimally selected sensor set SS1 is replaced with S31:+Y. Large differences are visible in the low frequencies region, especially between $0 \mathrm{~Hz}$ and $20 \mathrm{~Hz}$.

5.4. The estimation is robust with respect to the covariance of the random walk model for the unknown inputs, when properly chosen above clearly identifiable values

Figure 23 shows inputs estimation results related to the WCLs for experimental run 4 of Table 2 which corresponds to the road profile reproduction. The estimated signals have been obtained by again making use of sensor set SS1, but this time several results for different values of parameter $Q_{u}$ are reported.

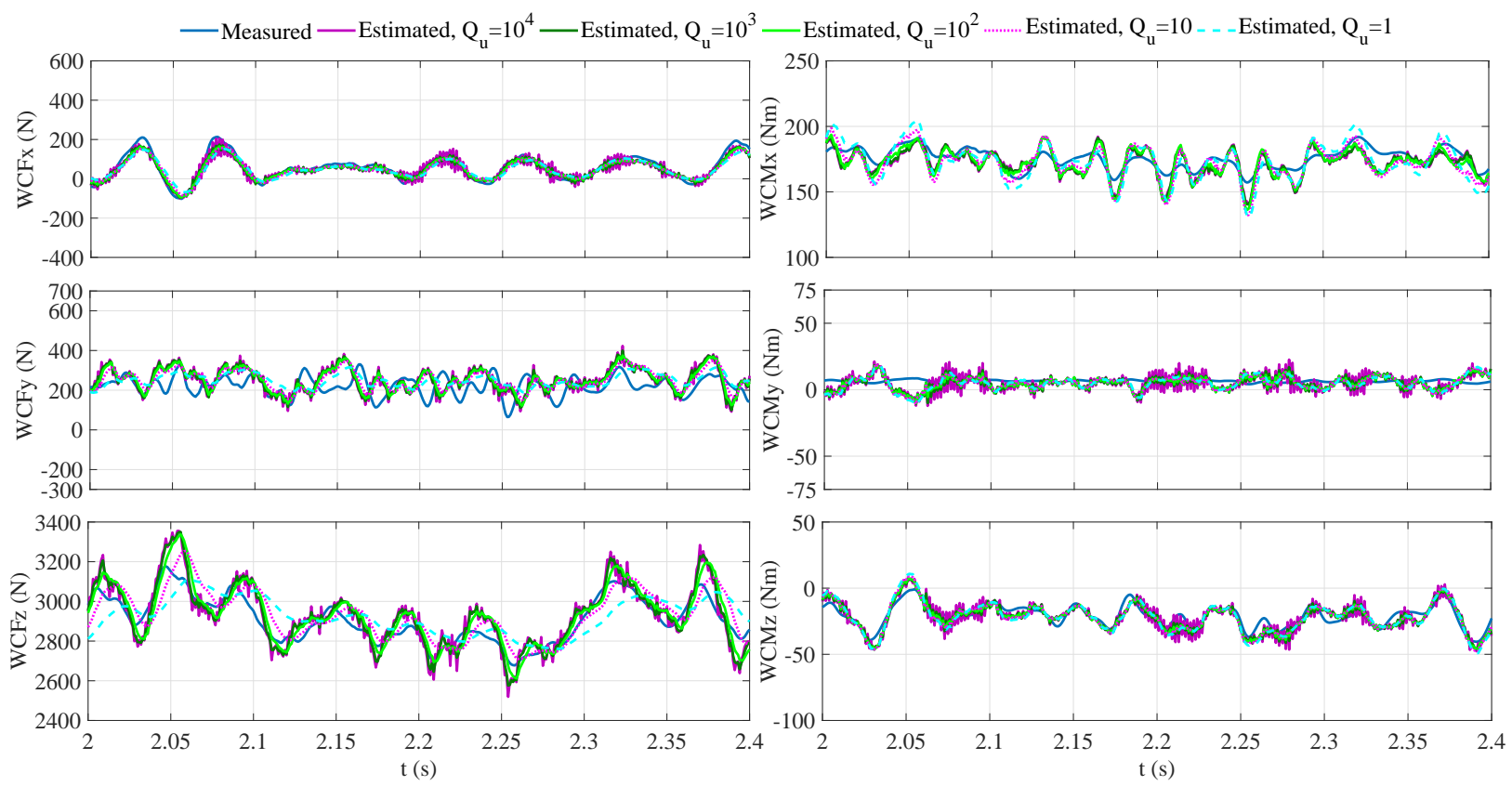

Figure 23: A value of $Q_{u}$, i.e. 10, can be easily identified, above which all estimation signals converge to the same result except for a small increase of additional noise.

It can be observed that variations of such parameter for several order of magnitudes have only a negligible impact on the estimation results for WCFx,WCFy,WCMx,WCMy and WCMz. WCFz is instead more affected by those variations. More in detail it can be observed how the lowest value of 1 implies a lower estimated amplitude and a certain delay in the signal reconstruction. The increase to 10 determines an increase in the estimated amplitude and considerable reduction on the delay. All estimation signals corresponding to $Q_{u} \geqslant 10$ finally seem to converge to the same result. The signals then only differ from each other by a small increase of noise for increasing values of $Q_{u}$.

This behavior, of better signal tracking and worse noise rejection with higher values of $Q_{u}$ and viceversa for lower values of $Q_{u}$, is typical for the AKF and has already been observed in a number of works including [8] and [43]. The same behavior for all other five loads would be observed if results for a wider spectrum of values of $Q_{u}$ were also reported. 
However it is clear that in the reported case a lower bound for of $Q_{u}$, i.e. 10, can be easily identified, above which all estimation signals converge to the same result except for a small increase of additional noise. This lower bound has been used in all estimation results of Subsections 5.1, 5.2 and 5.3.

\section{Conclusions}

In this work, a general procedure has been presented to derive an augmented extended Kalman filter for systems described by an implicit state equation of quite general form. The implicit nature of the method allows to easily employ implicit time-discretization schemes that are needed to cope with the numerical stiffness arising in the resolution of certain classes of mechanical problems, e.g. the time integration of flexible multibody systems. Analytical relations have been obtained to compute the linearization of the implicit system needed to apply the estimation scheme, starting from the tangent matrices of the system. This provided the means for a computationally efficient estimation procedure, which is a clear advantage. On the other hand, as in any other extended Kalman filter based approach, it might be necessary to considerably reduce the time step size in the cases in which the system undergoes strong non-linearities, which instead represents a disadvantage.

The methodology has been applied to the case of estimation of the six wheel center loads in a vehicle suspension test rig based on a McPherson suspension, which is closely related to the industrial case of measurement of input quantities for durability assessments in automotive. In order to accurately reconstruct the time signals of all six loads a flexible multibody model of the system has been employed. This also allowed to estimate the full strain field on the suspension components.

The results showed that when the model employed in the estimation procedure incorporated accurate input-output relations between the loads to be estimated and the measured quantities, accurate estimations of all six loads and of the full strain field on the suspension components could be carried out. When instead such input-output relations were not accurate, the full strain field was still accurately estimated while the accuracy of the estimated loads degraded. Moreover it has been shown how the simulation model availability enables to optimally place the sensors in order to observe all the quantities of interest, as an observability requirement also has to be satisfied.

\section{Acknowledgements}

The authors gratefully acknowledge the European Commission for its support of the Marie Sklodowska Curie program through the ITN ANTARES project (GA 606817), the support of VLAIO (Flemish Innovation \& Entrepreneurship) through the O\&O project ENDURANCE (IWT 150958) and through the Innovation Mandate project VIRTUAL MATES (IWT 140778), and the support of the Research Fund KU Leuven. The authors also acknowledge Daniele Brandolisio for his support in the execution of the experimental activity.

\section{References}

[1] P. Johannesson, M. Speckert, Guide to Load Analysis for Durability in Vehicle Engineering, John Wiley and Sons, Ltd., Chichester, West Sussex, United Kingdom, 2014.

[2] H. Van Der Auweraer, T. Tamarozzi, E. Risaliti, M. Sarrazin, J. Croes, B. Forrier, F. Naets, W. Desmet, Virtual Sensing Based on Design Engineering Simulation Models, in: proc. of the International Conference on Structural Engineering Dynamics, ICEDyn (2017).

[3] S. Gillijns, B. De Moor, System inversion with application to filtering and smoothing in the presence of unknown inputs, Technical Report ESAT-SISTA/TR 07-08, KU Leuven, 2007.

[4] L. J. Nordstrom, T. Nordberg, A time delay method to solve non-collocated input estimation problems, Mechanical Systems and Signal Processing 18 (2004) 1469-1483.

[5] D. Bernal, A. Ussia, Sequential deconvolution input reconstruction, Mechanical Systems and Signal Processing 50-51 (2015) $41-55$.

[6] M. V. van der Seijs, D. de Klerk, D. J. Rixen, General framework for transfer path analysis: History, theory and classification of techniques, Mechanical Systems and Signal Processing 68-69 (2016) 217-244.

[7] R. Kalman, A new approach to linear filtering and prediction problems, Journal of Basic Engineering 82 (1960) $35-45$.

[8] E. Lourens, E. Reynders, G. De Roeck, G. Degrande, G. Lombaert, An augmented Kalman filter for force identification in structural dynamics, Mechanical Systems and Signal Processing 27 (2012) 446-460.

[9] F. Naets, J. Croes, W. Desmet, An online coupled state/input/parameter estimation approach for structural dynamics, Computer Methods in Applied Mechanics and Engineering 283 (2015) 1167-1188.

[10] F. Naets, J. Cuadrado, W. Desmet, Stable force identification in structural dynamics using Kalman filtering and dummy-measurements, Mechanical Systems and Signal Processing 50-51 (2015) 235-248. 
[11] E.A. Wan, A.T. Nelson, Dual extended Kalman filter methods, in: S. Haykin (Ed.), Kalman Filtering and Neural Networks, Wiley, New York, 2001, pp. 123-173.

[12] S. Eftekhar Azam, E. Chatzi, C. Papadimitriou, A dual Kalman filter approach for state estimation via output-only acceleration measurements, Mechanical Systems and Signal Processing 60-61 (2015) 866-886.

[13] S. Gillijns, B. De Moor, Unbiased minimum-variance input and state estimation for linear discrete-time systems, Automatica 43 (2007) $111-116$.

[14] S. Gillijns, B. De Moor, Unbiased minimum-variance input and state estimation for linear discrete-time systems with direct feedthrough, Automatica 43 (2007) 934-937.

[15] E. Lourens, C. Papadimitriou, S. Gillijns, E. Reynders, G. De Roeck, G. Lombaert, Joint input-response estimation for structural systems based on reduced-order models and vibration data from a limited number of sensors, Mechanical Systems and Signal Processing 29 (2012) 310-327.

[16] K. Maes, A. Smyth, G. De Roeck, G. Lombaert, Joint input-state estimation in structural dynamics, Mechanical Systems and Signal Processing 70-71 (2016) 445-466.

[17] D. Simon, Optimal state estimation: Kalman, $\mathrm{H}_{\infty}$ and nonlinear approaches, Wiley-Interscience, Hoboken, NJ, 2006.

[18] S. Julier, J. Uhlmann, H. F. Durrant-Whyte, A new method for the nonlinear transformation of means and covariances in filters and estimators, IEEE Transactions on Automatic Control 45 (2000) 477-482.

[19] J. J. LaViola, A Comparison of Unscented and Extended Kalrnan Filtering for Estimating Quaternion Motion, in: proc. of the American Control Conference (2003)

[20] W. Song, Generalized minimum variance unbiased joint input-state estimation and its unscented scheme for dynamic systems with direct feedthrough, Mechanical Systems and Signal Processing 99 (2018) 886-920.

[21] J. Cuadrado, D. Dopico, A. Barreiro, E. Delgado, Real-time state observers based on multibody models and the extended Kalman filter, Journal of mechanical science and technology 23 (2009) 894-900.

[22] R. Pastorino, D. Richiedei, J. Cuadrado, A. Trevisani, State estimation using multibody models and non-linear Kalman filters, International Journal of Non-Linear Mechanics 53 (2013) 83-90.

[23] F. Naets, R. Pastorino, J. Cuadrado, W. Desmet, Online state and input force estimation for multibody models employing extended Kalman filtering, Multibody System Dynamics 32 (2014) 317-336.

[24] I. Palomba, D. Richiedei, A. Trevisani, Two-stage approach to state and force estimation in rigid-link multibody systems, Multibody System Dynamics 39 (2017) 115-134.

[25] E. Risaliti, M. Vermaut, J. Croes, B. Cornelis, W. Desmet, Force estimation on a McPherson system by means of a state estimator and a multibody model, in: proc. of the ECCOMAS Thematic Conference on Multibody Dynamics (2017).

[26] E. Sanjurjo, D. Dopico, A. Luaces, M. n. Naya, State and force observers based on multibody models and the indirect Kalman filter, Mechanical Systems and Signal Processing 106 (2018) 210-228.

[27] E. Hairer, G. Wanner, Solving Ordinary Differential Equations II. Stiff and Differential-Algebraic Problems, volume 14 of Springer Series in Computational Mathematics, Springer-Verlag Berlin Heidelberg, Heidelberg, 1996.

[28] J. B. Jorgensen, P. G. Thomsen, H. Madsen, M. R. Kristensen, A Computationally Efficient and Robust Implementation of the ContinuousDiscrete Extended Kalman Filter, in: proc. of the American Control Conference (2007).

[29] T. Mazzoni, Computational aspects of continuous discrete extended Kalman-filtering, Computational Statistics 23 (2008) 519-539.

[30] M. Kulikova, G. Kulikov, Adaptive ODE solvers in extended Kalman filtering algorithms, Journal of Computational and Applied Mathematics 262 (2014) 205-216.

[31] A. A. Shabana, Euler parameters kinetic singularity, Proceedings of the Institution of Mechanical Engineers, Part K: Journal of Multi-body Dynamics 228 (2014) 307-313.

[32] M. Vermaut, F. Naets, W. Desmet, A Flexible Natural Coordinates Formulation (FNCF) for the efficient simulation of small-deformation multibody systems, International Journal for Numerical Methods in Engineering (2018).

[33] A. Gryzlov, M. Leskens, R. Mudde, A semi-implicit approach for fast parameter estimation by means of the extended Kalman filter, Journal of Process Control 21 (2011) 510-518.

[34] A. Peirce, F. Rochinha, An integrated extended Kalman filter implicit level set algorithm for monitoring planar hydraulic fractures, Inverse Problems 28 (2012) 015009.

[35] J. G. De Jalon, E. Bayo, Kinematic and dynamic simulation of multibody systems: the real-time challenge, Springer Science \& Business Media, 2012.

[36] O. C. Zienkiewicz, R. L. Taylor, The finite element method, Butterworth Heinemann, Oxford, 2000.

[37] B. Besselink, U. Tabak, A. Lutowska, N. van de Wouw, H. Nijmeijer, D. Rixen, M. Hochstenbach, W. Schilders, A comparison of model reduction techniques from structural dynamics, numerical mathematics and systems and control, Journal of Sound and Vibration 332 (2013) 4403-4422.

[38] R. R. Craig, A review of time-domain and frequency domain component mode synthesis methods, Journal of Modal Analysis 2 (1987) 59-72.

[39] International Standard ISO 8608: Mechanical vibration - Road surface profiles - Reporting of measured data, 2016.

[40] T. Tamarozzi, E. Risaliti, W. Rottiers, K. Janssens, W. Desmet, Noise, ill-conditioning and sensor placement analysis for force estimation through virtual sensing, in: proc. of the International Conference on Noise and Vibration Engineering, ISMA (2016).

[41] W. Rottiers, F. Naets, B. Forrier, M. Sarrazin, H. Van Der Auweraer, W. Desmet, Mechatronic powertrain virtual sensor: system-level model and sensor selection, in: proc. of the International Conference on Structural Engineering Dynamics, ICEDyn (2017).

[42] B. K. Ghosh, J. Rosenthal, A generalized Popov-Belevitch-Hautus test of observability, IEEE Transactions on Automatic Control 40 (1995) $176-180$.

[43] E. Risaliti, J. Van Cauteren, T. Tamarozzi, B. Cornelis, W. Desmet, Virtual sensing of wheel center forces by means of a linear state estimator, in: proc. of the International Conference on Noise and Vibration Engineering, ISMA (2016). 


\section{Appendix A. Measurement equation for strain gauges}

In this work strain gauges are used in combination with a flexible multibody model in the estimation procedure exposed in Section 2. In this appendix, how to retrieve the necessary function $\mathbf{h}$ related to the measurement equation for strain measurements and the relative linearization matrix $\mathbf{H}$ is described.

As explained in Section 3, the steering knuckle and the control arm are the suspension components on which the strain gauges are instrumented. Furthermore these components are modeled as reduced order linear finite element models as explained in the same section. The finite element formulation is used here to obtain function $\mathbf{h}$ and matrix H.

$\mathbf{h}$ is a vectorial function in which each component is related to a certain measurement. The relative component function $h^{j}$ for strain gauge $j$ attached to body $i$ can be obtained by considering that the elongation $\varepsilon_{n^{j}}$ along the direction of strain gauge $j$ is given by

$$
\varepsilon_{n^{j}}=\mathbf{n}^{j T} \varepsilon^{j} \mathbf{n}^{j}
$$

which represents the projection of the strain tensor $\varepsilon^{j}$ at the strain gauge location onto the strain gauge direction identified by versor $\mathbf{n}^{j}$. If the strain tensor components relative to a reference frame $\mathcal{S}_{i}$ attached to body $i$ are arranged in the column vector $\bar{\varepsilon}_{n^{j}}$ as

$$
\bar{\varepsilon}_{n^{j}}=\left[\begin{array}{llllll}
\varepsilon_{11} & \varepsilon_{22} & \varepsilon_{33} & \varepsilon_{12} & \varepsilon_{23} & \varepsilon_{31}
\end{array}\right]^{T}
$$

and if moreover the direction cosines of $\mathbf{n}^{j}$ related to $\mathcal{S}_{i}$ are arranged in the column vector $\overline{\mathbf{n}}^{j}$ as follows

$$
\overline{\mathbf{n}}^{j}=\left[\begin{array}{llllll}
n_{1}^{2} & n_{2}^{2} & n_{3}^{2} & n_{1} n_{2} & n_{2} n_{3} & n_{3} n_{1}
\end{array}\right]^{T}
$$

the elongation $\varepsilon_{n^{j}}$ can then be computed as

$$
\varepsilon_{n^{j}}=\overline{\mathbf{n}}^{j T} \bar{\varepsilon}_{n^{j}}
$$

By means of the finite element formulation, it is then possible to write vector $\bar{\varepsilon}_{n^{j}}$ in terms of the nodal displacement vector $\mathbf{z}^{i}$ for body $i[36]$ as

$$
\bar{\varepsilon}_{n^{j}}=\mathbf{N z}^{i}
$$

where $\mathbf{N}$ is a matrix of appropriate dimensions which contains spatial derivatives of the shape functions and hence depends on the specific finite elements that build up the model. Due to the model order reduction applied to each body then, the nodal displacement vector $\mathbf{z}$ for body $i$ can be approximated as

$$
\mathbf{z}^{i}=\boldsymbol{\Psi}^{i} \mathbf{q}_{f}^{i}
$$

where $\boldsymbol{\Psi}^{i}$ and $\mathbf{q}_{f}^{i}$ are the matrix of the reduction basis modes and the vector of the model participation factors for body $i$ introduced in Section 3. Finally by combining Eqs. A.4, A.5 and A.6 the following relation can be obtained

$$
\varepsilon_{n^{j}}=\overline{\mathbf{n}}^{j T} \mathbf{N} \boldsymbol{\Psi}^{i} \mathbf{q}_{f}^{i}=\overline{\mathbf{n}}^{j T} \mathbf{N} \boldsymbol{\Psi}^{i} \mathbf{C}^{i} \mathbf{x}
$$

where the Boolean matrix $\mathbf{C}^{i}$ of appropriate dimensions has been also introduced which selects the flexible coordinates of body $i$ from the state vector $\mathbf{x}$. From Eqs. A.7 and 26 then it follows that

$$
h^{j}(\mathbf{x})=\overline{\mathbf{n}}^{j T} \mathbf{N} \Psi^{i} \mathbf{C}^{i} \mathbf{x}, \quad \mathbf{H}^{j}=\overline{\mathbf{n}}^{j T} \mathbf{N} \Psi^{i} \mathbf{C}^{i}
$$

where $\mathbf{H}^{j}$ is the row of the Jacobian matrix $\mathbf{H}$ associated to $h^{j}$. By stacking $h^{j}$ and $\mathbf{H}^{j}$ related to all the included sensors, the complete vector $\mathbf{h}$ and matrix $\mathbf{H}$ can be found. It can be noticed from Eq. A. 8 that in the case where small deformations and a linear relationship as in Eq. A.5 are assumed, $\mathbf{h}$ is linear and $\mathbf{H}$ is constant with respect to $\mathbf{x}$ for strain measurements. 